\author{
RESEARCH ARTICLE \\ 10.1029/2020JC016977 \\ Key Points: \\ - In situ measurements are used to \\ estimate ice-ocean drag across a \\ wide range of ice conditions based \\ on the sea ice momentum balance \\ - Ice-ocean drag coefficients show \\ a seasonal cycle with a spring \\ maximum and a fall minimum, \\ following the growth and melt of \\ ice keels \\ - Geometry-based drag \\ parameterization schemes are able \\ to capture much of the observed \\ variability using direct ice geometry \\ measurements
}

Supporting Information: Supporting Information may be found in the online version of this article.

Correspondence to:

S. Brenner,

sdbren@uw.edu

Citation:

Brenner, S., Rainville, L., Thomson, J., Cole, S., \& Lee, C. (2021). Comparing observations and parameterizations of ice-ocean drag through an annual cycle across the Beaufort Sea. Journal of Geophysical Research: Oceans, 126, e2020JC016977. https://doi. org/10.1029/2020JC016977

Received 13 NOV 2020 Accepted 18 MAR 2021

\section{Comparing Observations and Parameterizations of Ice-Ocean Drag Through an Annual Cycle Across the Beaufort Sea}

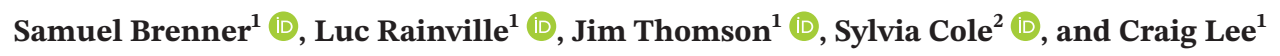 \\ ${ }^{1}$ Applied Physics Laboratory, University of Washington, Seattle, WA, USA, ${ }^{2}$ Woods Hole Oceanographic Institution, \\ Woods Hole, MA, USA
}

Abstract Understanding and predicting sea ice dynamics and ice-ocean feedback processes requires accurate descriptions of momentum fluxes across the ice-ocean interface. In this study, we present observations from an array of moorings in the Beaufort Sea. Using a force-balance approach, we determine ice-ocean drag coefficient values over an annual cycle and a range of ice conditions. Statistics from high resolution ice draft measurements are used to calculate expected drag coefficient values from morphology-based parameterization schemes. With both approaches, drag coefficient values ranged from $\sim 1$ to $10 \times 10^{-3}$, with a minimum in fall and a maximum at the end of spring, consistent with previous observations. The parameterizations do a reasonable job of predicting the observed drag values if the under ice geometry is known, and reveal that keel drag is the primary contributor to the total ice-ocean drag coefficient. When translations of bulk model outputs to ice geometry are included in the parameterizations, they overpredict drag on floe edges, leading to the inverted seasonal cycle seen in prior models. Using these results to investigate the efficiency of total momentum flux across the atmosphereice-ocean interface suggests an inter-annual trend of increasing coupling between the atmosphere and the ocean.

Plain Language Summary Sea ice moves in response to the push and pull (a.k.a., "drag") of both wind and ocean currents, so speeds of both the ice and the underlying ocean depends on how efficient that drag is. By looking at measurements of ice motion in response to the wind and ocean currents from three sites in the Beaufort Sea, we have calculated drag efficiency over 1 year. Computer models predict drag efficiency based on how rough the bottom of the sea ice is. Our measurements of the shape of the sea ice bottom are used to test and verify the framework for calculating drag efficiency that is in place in those models. The model framework can do a reasonable job of prediction if given good measurements of how rough the ice is, but may not be good at predicting that roughness. Because of that, current models might overpredict the drag efficiency while ice is melting. With our measurements of drag efficiency, we calculate how the sea ice impacts the total ability of the wind to push on the ocean and find that it is enhanced by the sea ice. As Arctic sea ice becomes more seasonal, we expect this enhancement to increase.

\section{Introduction}

Ongoing and dramatic changes in Arctic sea ice (e.g., Stroeve \& Notz, 2018) and the underlying ocean (Armitage et al., 2020; Jackson et al., 2011; Timmermans et al., 2018) highlight the need to understand Arctic system feedback processes. Sea ice dynamics are thought to play an important role in both localized (e.g., Ivanov et al., 2016) and large-scale ice-ocean feedbacks (Armitage, Manucharyan, et al., 2020; Dewey et al., 2018; Meneghello et al., 2018). However, there are still fundamental gaps in our knowledge of the role of sea ice in mediating momentum transfer across the atmosphere-ice-ocean system, especially in understanding spatial and seasonal variability in ice-ocean drag.

Turbulent processes in the ocean and in the atmosphere drive surface momentum flux (a.k.a., stress, $\tau$ ) across the ice-ocean and ice-atmosphere interfaces. These turbulent fluxes are commonly described by the quadratic drag law:

$$
\tau=\rho C u|u|
$$


which uses a turbulent transfer coefficient (or drag coefficient), $C$, to describe the momentum flux, $\tau$, in terms of an appropriate bulk, velocity $\boldsymbol{u}$. Thus, the ice-ocean stress, $\boldsymbol{\tau}_{i o}$, and atmosphere-ice stress, $\boldsymbol{\tau}_{a i}$ depend on ice-ocean and atmosphere-ice drag coefficients: $C_{i o}$ and $C_{a i}$, respectively. While there has been considerable work in relating observed values of the atmosphere-ice drag coefficient, $C_{a i}$, to sea ice properties (Andreas, 2011; Andreas, Horst, et al., 2010a; Arya, 1975; Castellani, Lüpkes, et al., 2014; Elvidge et al., 2016; Garbrecht et al., 2002; Guest \& Davidson, 1987; Lüpkes \& Birnbaum, 2005; Lüpkes, Gryanik, Hartmann, \& Andreas, 2012; Petty et al., 2017, and others), there is relatively little analogous work on the ice-ocean drag coefficient, $C_{i o}$. Indeed, despite a wide range of observed values of $C_{i o}$ spanning across an order of magnitude (e.g., Cole, Timmermans, et al., 2014; Cole, Toole, et al., 2017; McPhee, 1980, 2002; Morison et al., 1987; Randelhoff et al., 2014; Shaw et al., 2008), by default many sea ice models use a constant value for the drag coefficient (e.g., Köberle \& Gerdes, 2003; Losch et al., 2010; Rampal et al., 2016; Rousset et al., 2015; Timmermann et al., 2009), such as the "canonical" value of $C_{i o}=5.5 \times 10^{-3}$ determined by McPhee (1980). Moreover, studies show that modeled sea ice thickness is sensitive to the chosen value of $C_{i o}$ (Hunke, 2010; J. G. Kim et al., 2006).

Recent observations show both spatial and seasonal variations in the ice-ocean drag coefficient (Cole, Toole, et al., 2017), suggesting the importance of ice morphology on the values of $C_{i o}$ (e.g., due to form drag; Lu et al., 2011; Steele, Morison, \& Untersteiner, 1989; Tsamados et al., 2014). Model studies that incorporate a variable ice-ocean drag via parameterization of form drag (directly, Tsamados et al., 2014; or indirectly, Steiner, 2001) show first-order impacts both on the sea ice (Castellani, Losch, et al., 2018) and the underlying ocean (Castellani, Gerdes, et al., 2015; Castellani, Losch, et al., 2018; Martin, Tsamados, et al., 2016). Although form drag parameterizations of the ice-ocean drag provide a nice theoretical description for the relationship between sea ice morphology and the ice-ocean drag coefficient (Lu et al., 2011; Tsamados et al., 2014), until now there has been no detailed observational study comparing morphological features with observed values of $C_{i o}$ across a range of sea ice conditions.

In this study, we present observations made over an annual cycle from an array of moorings in the Beaufort Sea. Using a force-balance approach, mooring measurements and atmospheric re-analysis data are used to infer ice-ocean drag coefficients. Uplooking sonar on the moorings provide snapshots of under-ice topography and statistics related to ice keels and floe edges. Together, these results (1) provide insight into the morphological drivers underlying variations of the ice-ocean drag coefficient, (2) are used for evaluation of model parameterization schemes, and (3) provide context for a broader understanding of momentum transfer into the upper ocean in the changing Arctic. The remainder of this paper is organized as follows: Sections 1.1 and 1.2 provide additional background about momentum fluxes across the atmosphere-ice-ocean interface (with focus on the sea ice momentum equation and the total atmosphere-ocean momentum flux). Section 2 provides a review of the geometry-based parameterization schemes developed by Lu et al. (2011) and Tsamados et al. (2014), thus giving important context for interpreting the study results. In Section 3 we describe the field study and measurements, along with the force-balance and geometry-based descriptions of the ice-ocean drag coefficient. Descriptions of variations in $C_{i o}$, along with evaluation of the parameterization schemes, and a description of the morphological drivers of ice-ocean drag are presented in Section 4. Then, in Section 5, these results are placed in the context of previous observations of ice-ocean drag and total momentum flux. The main contributions of the study are summarized in Section 6.

\subsection{The Sea Ice Momentum Equation}

The conservation of momentum of sea ice can be written as (e.g., Heorton, Feltham, \& Hunt, 2014):

$$
m_{e}[\underbrace{\frac{\partial \boldsymbol{u}_{i}}{\partial t}}_{\mathrm{I}}+\underbrace{\boldsymbol{u}_{i} \cdot \nabla \boldsymbol{u}_{i}}_{\mathrm{II}}+\underbrace{f \hat{\boldsymbol{k}} \times \boldsymbol{u}_{i}}_{\mathrm{III}}]=\underbrace{A \tau_{a i}}_{\mathrm{IV}}+\underbrace{A \tau_{o i}}_{\mathrm{V}}+\underbrace{\nabla \cdot \sigma}_{\mathrm{VI}}+\underbrace{m_{e} g \nabla \eta}_{\mathrm{VII}},
$$

for $m_{e}$ the "effective" ice mass per unit area, $m_{e}=A \rho_{i} h_{i}$, and other variables as defined in Table 1 , with $\nabla$ the horizontal gradient operator. This form of the sea ice momentum equation is consistent with that presented by Leppäranta (2011), but modified to ensure consistent scaling for mixed ice-open water conditions 
Table 1

Notation

\begin{tabular}{|c|c|}
\hline$a_{i}$ & Ice covered area \\
\hline$a_{r d g}$ & Area covered in ridged ice \\
\hline$b_{1}, b_{2}, A_{*}$ & Geometry parameters \\
\hline$A$ & Ice concentration \\
\hline$c_{f}$ & Floe-edge drag coefficient of resistance \\
\hline$c_{k}$ & Keel drag coefficient of resistance \\
\hline$c_{s}$ & Skin drag coefficient of resistance \\
\hline$C$ & Generic drag coefficient \\
\hline$C_{f}$ & Form drag from floe edges \\
\hline$C_{k}$ & Form drag from keels \\
\hline$C_{s}$ & Skin drag \\
\hline$C_{a o}$ & Atmosphere-ocean drag coefficient \\
\hline$C_{a i}$ & Atmosphere-ice drag coefficient \\
\hline$C_{i o}$ & Ice-ocean drag coefficient \\
\hline$C_{\text {equiv }}$ & Atmosphere-ocean equivalent drag \\
\hline$d_{i}$ & Ice draft \\
\hline$d_{l v l}$ & Level ice draft \\
\hline$f$ & Coriolis parameter \\
\hline $\boldsymbol{F}_{a}$ & Ice acceleration force \\
\hline $\boldsymbol{F}_{i}$ & Ice interaction force \\
\hline$g$ & Gravitational acceleration \\
\hline$h_{i}$ & Ice thickness \\
\hline$h_{k}$ & Keel depth (generic) \\
\hline$h_{k \mathrm{rel}}$ & Relative keel depth \\
\hline$h_{k \text { tot }}$ & Total keel depth \\
\hline$\ell_{f}$ & Floe length \\
\hline$\ell_{k}$ & Keel spacing \\
\hline$e_{l}$ & Lead length \\
\hline$m_{e}$ & Effective ice mass per unit area \\
\hline$m_{w}$ & Skin drag attenuation parameter \\
\hline$P_{0}$ & Boundary-layer integration function \\
\hline$S_{c}$ & Sheltering function \\
\hline$s_{l}$ & Attenuation parameter \\
\hline$u$ & Generic bulk velocity \\
\hline $\boldsymbol{u}_{*}$ & Friction velocity \\
\hline $\boldsymbol{u}_{a}$ & Wind velocity at $10 \mathrm{~m}$ \\
\hline $\boldsymbol{u}_{i}$ & Ice drift velocity \\
\hline $\boldsymbol{u}_{o}$ & Ocean velocity at a reference depth \\
\hline $\boldsymbol{u}_{g}$ & Geostrophic ocean velocity \\
\hline $\boldsymbol{u}_{\text {rel }}$ & Ice-ocean relative velocity \\
\hline$v_{r d g}$ & Volume of ridged ice \\
\hline$z_{0}$ & Roughness length \\
\hline$z_{0 i}$ & Level ice bottom roughness length \\
\hline
\end{tabular}

(per Connolley et al., 2004; Hunke \& Dukowicz, 2003). The terms of the equation are as follows: (I) local ice acceleration; (II) advective ice acceleration; (III) Coriolis acceleration; (IV) stress of the atmosphere acting on the ice; (V) stress of the ocean acting on the ice; (VI) internal stress ("ice-ice" stress); and (VII) gravitational force from sea surface tilt. Advective acceleration (term II) is generally considered negligible and excluded. The final term (VII) in Equation 2 can be expressed in terms of the geostrophic balance $f \hat{\boldsymbol{k}} \times \boldsymbol{u}_{g}=g \nabla \eta$ and then combined with the Coriolis term, so that term III becomes $f \hat{\boldsymbol{k}} \times\left(\boldsymbol{u}_{i}-\boldsymbol{u}_{g}\right)$ (Leppäranta, 2011). An additional term representing wave radiation stress in the marginal ice zone has been shown to be locally important at the ice edge (e.g., Perrie \& Hu, 1997; Steele, Morison, \& Untersteiner, 1989; Thomson et al., 2021), but overall is small, so it is neglected. Leppäranta (2011) also includes an atmospheric pressure gradient term which is not included here. In mixed ice-open water conditions, the ocean-ice and atmosphere-ice stresses $\left(\tau_{a i}\right.$ and $\tau_{o i}$ ) represent the stress acting only on the ice-covered area and are distinct from the total stress out of the ocean/atmosphere (Hunke \& Dukowicz, 2003).

Sea ice is considered to be in "free drift" if the internal ice stress (term VI) is negligible (e.g., Connolley et al., 2004; Hunke \& Dukowicz, 2003; Leppäranta, 2011; McPhee, 1980). This is often assumed to be the case if the ratio of ice speed to wind speed $\left(\left|\boldsymbol{u}_{i}\right| /\left|\boldsymbol{u}_{a}\right|\right.$, the "wind factor") is sufficiently high (typically $\geq 2 \%$; e.g., McPhee, 1980), or if ice concentration is sufficiently low (e.g., $\leq 85 \%$; Heorton, Tsamados, et al., 2019; Hunke $\&$ Dukowicz, 2003). For freely drifting sea ice, the ice-ocean stress $\left(\tau_{i o}=\right.$ $\left.-\tau_{o i}\right)$ can be expressed as:

$$
\boldsymbol{\tau}_{i o}=\boldsymbol{\tau}_{a i}-\rho_{o} d_{i}\left[\frac{\partial \boldsymbol{u}_{i}}{\partial t}+f \hat{\boldsymbol{k}} \times\left(\boldsymbol{u}_{i}-\boldsymbol{u}_{g}\right)\right],
$$

where the sea ice mass per unit area $\rho_{i} h_{i}$ (for ice density $\rho_{i}$ and total ice thickness $h_{i}$ ) has been replaced with $\rho_{0} d_{i}$ (for ocean density $\rho_{o}$ and ice draft $d_{i}$ ) assuming hydrostatic balance. McPhee (1980) and Dewey (2019) use this balance, assuming steady-state $\left(\frac{\partial \boldsymbol{u}_{i}}{\partial t}=0\right)$, in order to calculate ice-ocean stress and infer the ice-ocean drag coefficient, while Randelhoff et al. (2014) employ this equation retaining the local acceleration. The ice-ocean stress is also frequently presented in terms of friction velocity, $\boldsymbol{u}_{*}$, defined by $\tau_{i o}=\rho_{o} \boldsymbol{u}_{*}\left|\boldsymbol{u}_{*}\right|$.

\subsection{Total Momentum Flux Into the Ocean}

Using the quadratic drag law (Equation 1), the ice-ocean stress, $\tau_{i 0}$, and atmosphere-ice stress, $\tau_{a i}$, are written as:

$$
\begin{gathered}
\boldsymbol{\tau}_{i o}=\rho_{o} C_{i o} \boldsymbol{u}_{r e l}\left|\boldsymbol{u}_{r e l}\right|, \\
\boldsymbol{\tau}_{a i}=\rho_{a} C_{a i} \boldsymbol{u}_{a}\left|\boldsymbol{u}_{a}\right|,
\end{gathered}
$$

where the ice-ocean stress uses the relative ice-ocean horizontal velocity, $\boldsymbol{u}_{r e l}=\boldsymbol{u}_{i}-\boldsymbol{u}_{o}$, as a bulk velocity, while the atmosphere-ice stress uses the wind speed, $\boldsymbol{u}_{a}$ (for other variable definitions, see Table 1 ). The atmosphere-ice stress is also sometimes written with an atmosphere-ice relative velocity $\boldsymbol{u}_{a}-\boldsymbol{u}_{i}$ as the bulk velocity (mirroring the use of relative velocity 


\begin{tabular}{lc} 
Table 1 & \\
Continued & \\
$z_{0 w}$ & Roughness length of water \\
$z_{\text {ref }}$ & Reference depth \\
$\alpha_{k}$ & Keel slope \\
$\beta$ & Turning angle \\
$\eta$ & Sea surface displacement \\
$\kappa$ & von Kármán constant (=0.41) \\
$\rho$ & Density \\
$\rho_{a}$ & Air density \\
$\rho_{i}$ & Ice density \\
$\rho_{o}$ & Ocean density \\
$\sigma$ & Internal ice stress tensor \\
$\tau$ & Surface stress \\
$\tau_{a i}$ & Atmosphere-ice stress \\
$\tau_{a o}$ & Atmosphere-ocean stress \\
$\tau_{i o}$ & Ice-ocean stress \\
$\tau_{o i}$ & Ocean-ice stress \\
$\tau_{o c n}$ & Total ocean stress \\
$\tau_{a t m}$ & Total atmosphere stress \\
$\phi_{k}$ & Keel porosity \\
\hline & \\
\hline
\end{tabular}

in the ice-ocean stress), but since typically $\boldsymbol{u}_{a} \gg \boldsymbol{u}_{i}$, the ice velocity is frequently neglected from Equation $4 \mathrm{~b}$. It is also common to include a rotation matrix in Equation 4a to account for unresolved Ekman turning in the boundary layer (if the velocity is expressed as a complex exponential, $\boldsymbol{u}=u+i v$, then this is achieved by multiplying Equation 4a by $e^{i \beta}$ for turning angle $\beta$ ). While also occasionally included in Equation $4 \mathrm{~b}$, the much greater boundary layer heights in the atmosphere compared to the ocean means that there typically is not unresolved Ekman turning there, so it is not necessary to include an equivalent rotation matrix for calculating the atmosphere-ice stress (note that even with no atmospheric turning, there can still be an offset in wind direction and ice drift direction due to other forces in the sea ice momentum equation, and this offset is also occasionally referred to as a turning angle). Under-ice Ekman layers are not a subject of the present study, so rotation matrix is not included in Equation 4a, but we will accept values of $\tau_{i o}$ that are not aligned with $\boldsymbol{u}_{r e l}$ that result from the presence of Ekman turning.

In mixed ice and open-water conditions, there is both a direct transfer of momentum between the atmosphere and the ocean, and an indirect transfer mediated by sea ice. It is common to represent these fluxes as combinations of the corresponding atmosphere-ice-ocean stresses weighted by sea ice concentration (e.g., Martin, Steele, \& Zhang, 2014; Martin, Tsamados, et al., 2016). Then, the total momentum flux into the ocean, $\tau_{\text {ocn }}$, and the total momentum flux out of the atmosphere $\tau_{a t m}$ can be represented as:

$$
\begin{gathered}
\boldsymbol{\tau}_{o c n}=A \boldsymbol{\tau}_{i o}+(1-A) \boldsymbol{\tau}_{a o}, \quad \text { and } \\
\boldsymbol{\tau}_{a t m}=A \boldsymbol{\tau}_{a i}+(1-A) \boldsymbol{\tau}_{a o}:
\end{gathered}
$$

where $A$ is sea ice concentration, and each of the stress components (ice-ocean: $\tau_{i o}$; atmosphere-ice: $\tau_{a i}$; atmosphere-ocean: $\tau_{a o}$ ) are described by the quadratic drag law with corresponding drag coefficients: $\tau_{a o}=\rho_{a-}$ $C_{a o} \boldsymbol{u}_{\boldsymbol{a}}\left|\boldsymbol{u}_{\boldsymbol{a}}\right|$, and $\tau_{i o}, \tau_{a i}$ from Equations $4 \mathrm{a}$ and $4 \mathrm{~b}$. As a first approximation, the atmosphere-ocean drag coefficient, $C_{a 0}$, can be described as a function of wind speed (e.g., Large \& Yeager, 2004). The atmosphere-ice drag coefficient, $C_{a i}$, is expected to depend on sea ice geometry in a similar way to the ice-ocean drag (Andreas, 2011; Lüpkes, Gryanik, Hartmann, \& Andreas, 2012; Tsamados et al., 2014); however, it is sometimes parameterized simply as a function of ice concentration, $A$ (see Text $\mathrm{S} 2$ ), or taken as a constant (then the equivalent total atmospheric drag coefficient is still a function of ice concentration per Equation $5 \mathrm{~b}$; see Elvidge et al. [2016] for additional details and a comparison of atmospheric drag coefficient relationships with sea ice concentration for a variety of models).

Combining Equations 2, 5a, and 5b leads to the expression:

$$
\boldsymbol{\tau}_{o c n}=\boldsymbol{\tau}_{a t m}+\boldsymbol{F}_{i}+\boldsymbol{F}_{a}
$$

where $\boldsymbol{F}_{i}$ is the ice interaction force (derived from the inclusion of term VI in Equation 2), and $\boldsymbol{F}_{a}$ is the equivalent force from the acceleration and tilt terms (terms I, III, and VII in Equation 2; i.e., the term in brackets in Equation 3). Equation 6 mirrors the expression from Martin, Steele, and Zhang (2014, their Equation 2), except for the inclusion of the equivalent forces from ice acceleration, $\boldsymbol{F}_{a}$, which they neglect.

In the scenario where the transfer of momentum is an overall flux from the atmosphere into the ocean, this equation can be interpreted to state that all of the momentum flux out of the atmosphere $\left(\tau_{a t m}\right)$ goes into either the ice $\left(\boldsymbol{F}_{i}+\boldsymbol{F}_{a}\right)$, or into the ocean $\left(\tau_{o c n}\right)$. Although, because of the vector summation in Equation 6 , both of $\boldsymbol{F}_{i}$ and $\boldsymbol{F}_{a}$ can either enhance or subtract from $\boldsymbol{\tau}_{a t m}$. Ice interaction is usually thought of as a momentum sink that opposes $\tau_{\text {atm }}$ (Martin, Steele, \& Zhang, 2014; Steele, Zhang, et al., 1997), but ice acceleration terms could potentially be an additional source of ocean momentum. 
To examine the effect of sea ice in mediating the total momentum flux from the atmosphere to the ocean, consider an "equivalent drag coefficient", $C_{\text {equiv }}$, based on the construction of a quadratic drag law between the wind speed and the total ocean stress; that is,

$$
C_{\text {equiv }}=\frac{\left|\tau_{\text {ocn }}\right|}{\rho_{a}\left|\boldsymbol{u}_{a}\right|^{2}} .
$$

$C_{\text {equiv }}$ does not have a clean analytic form, nor is it a useful prognostic variable: its value will depend on $\boldsymbol{u}_{i}$ and $\boldsymbol{u}_{o}$, which are themselves functions of the total atmosphere-ice-ocean momentum transfer. Instead, $C_{\text {equiv }}$ is a diagnostic of momentum transfer efficiency, where higher values indicate that a greater proportion of atmospheric momentum is ultimately transferred to the ocean. This is similar to the use of a normalized effective stress in Martin, Steele, and Zhang (2014) and Martin, Tsamados, et al. (2016).

\section{Drag From Geometry-Based Parameterizations}

This study compares estimates of the observed ice-ocean drag to two schemes that parameterize the iceocean drag as a function of the observable ice geometry. Both Lu et al. (2011) and Tsamados et al. (2014) present similar ice geometry-based parameterizations of the ice-ocean drag coefficient based on a combination of skin and form drag components, with the scheme by Tsamados et al. (2014) available in the CICE sea ice model (Hunke et al., 2020). Steiner (2001) presents an alternative scheme using a "deformation energy" approach. That method has been used in the sea ice component of the MITgcm model (Losch et al., 2010) to investigate the impact of variable ice-ocean drag (Castellani, Losch, et al., 2018); however, we cannot track deformation energy with our measurements, so the deformation energy scheme is not considered here. Strictly, ice geometry-based parameterizations in the forms presented below only model the neutral ice-ocean drag coefficients and do not account for the impacts of stabilizing or destabilizing buoyancy fluxes. Buoyancy fluxes modify the total drag, and are included in atmospheric models as correction term to the neutral drag coefficient, based on Monin-Obukhov similarity theory (Monin \& Obukhov, 1954) (which could be included in sea ice models using a parameterization by Lüpkes \& Gryanik, 2015). We are unable to account for stability effects in the present study, which may modify some interpretations of the results.

\subsection{Details of Parameterization Schemes}

Ice-geometry based parameterizations of the ice-ocean drag coefficient write the total drag as a sum of form drag from floe edges, form drag from keels, and skin drag (Lu et al., 2011; Tsamados et al., 2014):

$$
C_{i o}=C_{f}+C_{k}+C_{s} .
$$

For both schemes, these three drag components can be written as:

$$
\begin{aligned}
& \text { floe edge drag: } \quad C_{f}=\frac{1}{2} c_{f} A \frac{d_{l v l}}{\ell_{f}}\left[S_{c}\left(\frac{d_{l v l}}{\ell_{l}}\right)\right]^{2} P_{0}\left(d_{l v l}, z_{0 w}\right), \\
& \text { keel drag: } \quad C_{k}=\frac{1}{2} c_{k} A \frac{h_{k}}{\ell_{k}}\left[S_{c}\left(\frac{h_{k}}{\ell_{k}}\right)\right]^{2} P_{0}\left(h_{k}, z_{0 i}\right), \\
& \text { skin drag: } \quad C_{s}=c_{s} A\left(1-m_{w} \frac{h_{k}}{\ell_{k}}\right), \quad \text { if } \frac{h_{k}}{\ell_{k}} \leq \frac{1}{m_{w}}
\end{aligned}
$$

with variables defined in Table 1 . So the ice geometry appears in the parameterizations as the floe "aspect ratio", $d_{l v l} / \ell_{f}$, and the "ridging intensity", $h_{k} / \ell_{k}$. The scheme by Tsamados et al. (2014) is an adaptation of an atmospheric drag parameterization by Lüpkes, Gryanik, Hartmann, and Andreas (2012). Note that in 


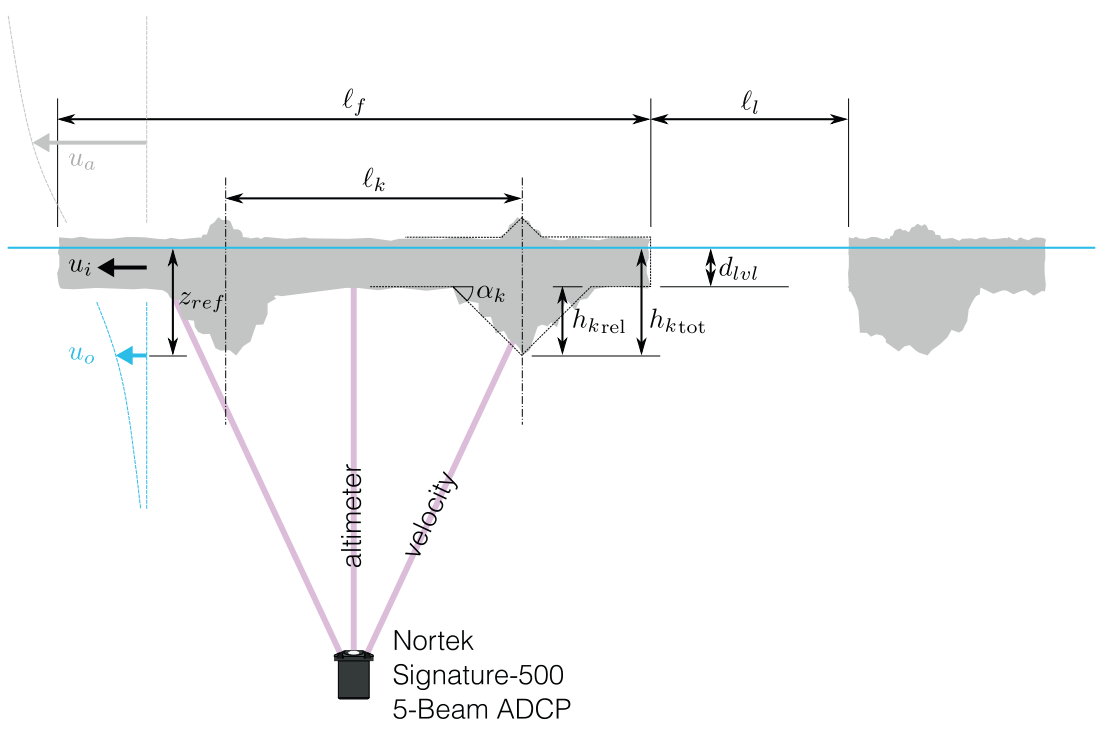

Figure 1. Schematic representation of an ice floe showing sea ice geometry with idealized triangular representation of ice keels, and the in situ ADCP measurements. Dimension labels of ice geometry correspond to Table 1.

Tsamados et al. (2014), the inequality in the valid range for the skin drag, $C_{s}\left(h_{k} / \ell_{k} \leq 1 / m_{w}\right)$, is mistakenly reversed (compare their Equation 19 with the work of Arya [1975] on which skin drag is based); Equation 9c presents the correct inequality for both of the parameterization schemes.

The two schemes are functionally similar. The differences between them are due to the following factors: (1) different values of the coefficients of resistance, $c_{f}, c_{k}$, and $c_{s}$ (which account for the drag on individual elements); (2) different forms of the "sheltering functions" $S_{c}$; and (3) the inclusion (or not) of the functions $P_{0}$ (which are included in the Tsamados et al. [2014] scheme but not in the Lu et al. [2011] scheme). Additionally, the two schemes use slightly different definitions for keel depth (relative vs. total; see Figure 1).

The sheltering function $S_{c}$ accounts for the reduction in drag of downstream obstacles due to the wake effect of upstream obstacles (Steele, Morison, \& Untersteiner, 1989). Both parameterization schemes employ different, empirically derived, sheltering functions:

$$
\begin{aligned}
\text { Tsamados et al. (2014) : } & S_{c}(x)=\left[1-\exp \left(-\frac{s_{l}}{x}\right)\right]^{1 / 2} \\
\text { Lu et al. (2011) : } & S_{c}(x)=\left[1-(x)^{1 / 2}\right]
\end{aligned}
$$

For keel sheltering, the input argument, $x$, is the ridging intensity, $h_{k} / \ell_{k}$, which mirrors its other use Equation $9 \mathrm{~b}$. For floe sheltering, the argument for the sheltering function is $d_{l v l} / \ell_{l}$ (the denominator is the distance between floes), instead of the aspect ratio $d_{l v l} / \ell_{f}$ that appears earlier in Equation 9a.

Tsamados et al. (2014) include a term in $C_{f}$ and $C_{k}$ which arises due to integration of a depth-varying velocity profile over the height of an obstacle, here called $P_{0}$ (it differs from the definition of $P_{0}$ in Lüpkes, Gryanik, Hartmann, and Andreas [2012]). In the atmospheric drag parameterization, Lüpkes, Gryanik, Hartmann, and Andreas (2012) assume a "law-of-the-wall" velocity profile: $u(z)=\left(u_{*} / \kappa\right) \ln \left(z / z_{0}\right)$, which Tsamados et al. (2014) maintains in adapting the scheme to the ice-ocean boundary layer. This gives

$$
P_{0}\left(h, z_{0}\right)=\left[\frac{\ln \left(h / z_{0}\right)}{\ln \left(z_{\text {ref }} / z_{0}\right)}\right]^{2},
$$


where the input variable $h$ is either the level ice draft, $d_{l v l}$ or keel depth, $h_{k}$ for floe edge drag or keel drag, respectively, and an appropriate choice of roughness length is used (see Equations $9 \mathrm{a}$ and $9 \mathrm{~b})$. Inclusion of $P_{0}$ allows the ice-ocean drag coefficient to be an explicit function of the reference depth $z_{\text {ref. }}$ The form of $P_{0}$ depends on the assumed law-of-the-wall boundary-layer structure, which is suitable for the atmosphere where the height of logarithmic boundary layer is typically much greater than the reference height $z_{\text {ref }}$ (e.g., Holton, 2004, chapter 5). However, it is not clear that this is appropriate in the ice-ocean boundary layer. The $P_{0}$ functions are not included in the scheme by Lu et al. (2011).

The coefficient of resistance, $c_{s}$ used in the skin drag parameterization ( $C_{s}$, Equation 9c) represents the baseline skin drag associated with level ice in the absence of ridges. Both Tsamados et al. (2014) and Lu et al. (2011) treat this term as a free parameter. Keeping with the law-of-the-wall velocity assumption used to develop $P_{0}$, the baseline skin drag could instead be represented by

$$
c_{s}=\left[\frac{\kappa}{\ln \left(z_{\mathrm{ref}} / z_{0 i}\right)}\right]^{2},
$$

where the von Kármán constant $\kappa=0.41$. This reduces the number of free parameters in the model, and allows $c_{s}$ to be an explicit function of the reference depth $z_{\text {ref. }}$ As with $P_{0}$, the actual form will depend strongly on boundary layer structure.

In applying their parameterization scheme (Equations 9, 10a and 11), Tsamados et al. (2014) use total keel depth, $h_{k \text { tot }}$, which is measured from the waterline (Figure 1) as the definition of $h_{k}$. However, in full ice cover, it should be the keel depth relative to the level ice draft, $h_{k \text { rel }}$, that contributes to form drag (as in Lu et al., 2011) (note: $h_{k \text { tot }}=h_{k \text { rel }}+d_{l v l}$ ). Similarly, the reference depth $z_{\text {ref }}$ in Equations 11 and 12 should also be relative to the level ice draft (e.g., $z_{\text {ref }}-d_{l v l}$ ), because that is the range over which the boundary layer develops. In mixed ice-open water conditions, the use of $h_{k \text { rel }}$ is still consistent with the parameterization scheme as floe-edge drag (Equation 9a) is accounted for separately.

\subsection{Translating Model Outputs to Ice Geometry}

The details of sea ice geometry necessary for calculating the ice-ocean drag coefficient with Equation 9 are not generally resolved by models, which do not simulate individual ice floes or keels. Tsamados et al. (2014) developed a scheme for estimating average keel properties based on outputs in the CICE model using assumptions about the keel geometry that are guided by observations (see their supplementary information). Namely, the scheme uses area extent and volume of ridged ice in a model grid cell ( $a_{r d g}$ and $v_{r d g}$, respectively), along with the ice area in a grid cell ( $a_{i}$, which is the ice concentration $A$ multiplied by the grid-cell area).

For subsurface measurements (as presented below), keel depth and keel spacing are given by taking the limit as $R_{h} \rightarrow \infty$ in Equations 24 and 25 from Tsamados et al. (2014) (where $R_{h}$ is the ratio of keel depth to sail height, so the limit states that all ridged ice in the measurements is attributed to keels). This gives the expressions:

$$
\begin{aligned}
& h_{k}=2 \frac{v_{r d g}}{a_{r d g}} \frac{b_{1}}{\phi_{k}}, \\
& \ell_{k}=2 h_{k} \frac{a_{i}}{a_{r d g}} \frac{b_{1}}{\tan \left(\alpha_{k}\right)},
\end{aligned}
$$

where $b_{1}$ is a weight function accounting for the overlap of keels with level ice (taken as 0.75 ), $\phi_{k}$ is the keel porosity (taken as 1), and $\alpha_{k}$ is the keel slope (see Figure 1).

The floe and lead lengths $\left(\ell_{f}, \ell_{l}\right)$ used in Equation 9a are also parameterized. Using measurements derived from aerial photographs of the marginal ice zone of Fram Strait, Lüpkes, Gryanik, Hartmann, and Andreas (2012) developed an empirical model for estimating floe size based on ice concentration: 


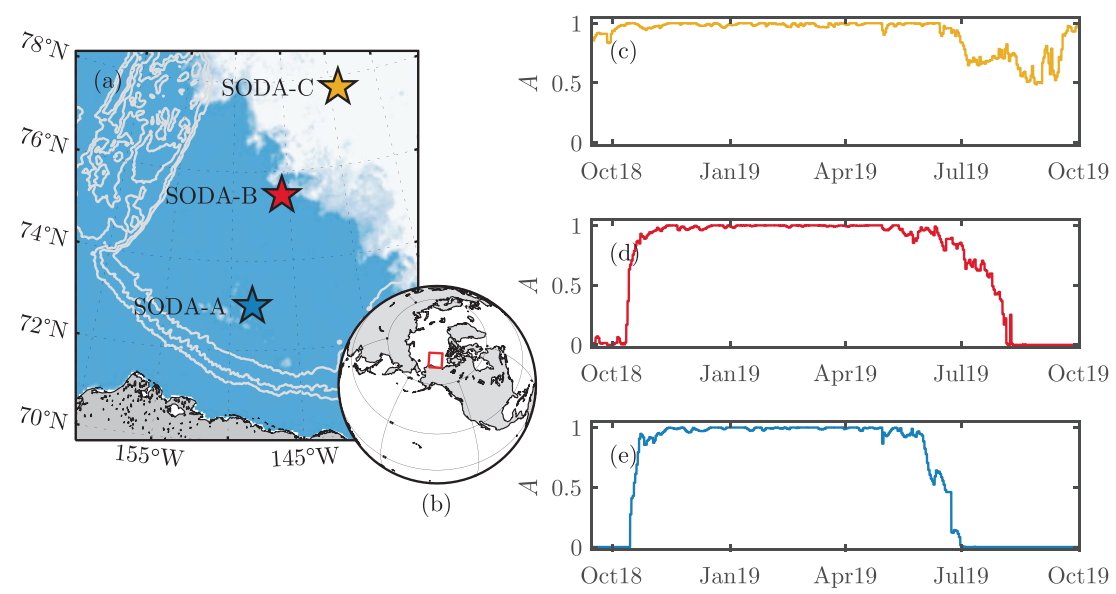

Figure 2. (a and b) Maps of (a) the Beaufort Sea showing the locations of the three moorings overlaid on sea ice concentration map from September 18, 2018 (the 2018 sea ice minimum), with bathymetry shown by gray contours (contours are 1000-m isobaths); and (b) the location of (a). The ice concentration in (a) is from the Sea Ice Remote Sensing database at the University of Bremen (Spreen et al., 2008). (c-e) The annual cycle of sea ice concentration averaged over the mooring locations during the measurement period: (c) SODA-C, (d) SODA-B, and (e) SODA-A.

$$
\ell_{f}=\ell_{f, \min }\left(\frac{A_{*}}{A_{*}-A}\right)^{b_{2}},
$$

with $b_{2}$ a tunable parameter (ranging from 0.3 to 1.4 ), and $A_{*}$ a value calculated such that the limits of $\ell_{f}$ range from $\ell_{f \text {,min }}$ to $\ell_{f \text {,max }}$ (for $A \rightarrow 0,1$ ), the minimum and maximum floe lengths, respectively (see Equation 27 in Lüpkes, Gryanik, Hartmann, and Andreas [2012]). Using default parameters, this gives average floe lengths that are limited to range from a minimum of $8 \mathrm{~m}$ to a maximum of $300 \mathrm{~m}$. Tsamados et al. (2014) implement this floe size model in their parameterization scheme, though they acknowledge that observations have shown that floe size follows a power-law distribution with a much wider range of scales than is possible with that scheme (e.g., Weiss \& Marsan, 2004; see also Stern et al. [2018] and references therein). They further acknowledge that this scheme may breakdown in the winter when ice concentration is near $100 \%$, given that the parameterization was developed for the marginal ice zone; but it is employed through the full year nonetheless.

\section{Drag From Field Measurements}

\subsection{Field Measurements}

Data were collected during the Stratified Ocean Dynamics of the Arctic (SODA) experiment: An Office of Naval Research (ONR) project to better understand the controls of heat and momentum transfer in the Arctic's upper ocean. A program component included the installation of three subsurface moorings in a line stretching from the south to the north of the Beaufort Sea, which are designated as SODA-A, SODA-B, and SODA-C (Figures 2a and 2b). The moorings recorded a full annual cycle of sea ice growth and melt from their installation in fall 2018 to their recovery in fall 2019. The spatial distribution of the moorings allowed for sampling of different ice regimes: the southernmost mooring (SODA-A) was in the seasonal ice zone and experienced prolonged open-water periods in summer (Figure 2e); SODA-B was near the edge of the seasonal ice zone and has a minimal open-water period but a longer period of time in marginal ice (Figure 2d); whereas SODA-C was still ice-covered all year long (Figure 2c; the mooring at that location was both deployed and recovered through the ice).

This study utilizes measurements made with uplooking Nortek Signature-500 5-beam acoustic Doppler current profilers (ADCPs) installed on the top float of each mooring (Figure 1). The instrument depths were $\sim 45 \mathrm{~m}$ for SODA-A, $42 \mathrm{~m}$ for SODA-B, and $27 \mathrm{~m}$ for SODA-C. To minimize the effects of mooring knockdown, the top float of each mooring was a DeepWater Buoyancy Stablemoor500, which are designed to 
remain level even during knockdown events (Harding et al., 2017). The maximum tilt deviation measured by any of the ADCPs was $\leq 2^{\circ}$ from their resting position. A Seabird SBE-37 conductivity-temperature-depth sensor installed underneath the float $(\sim 1 \mathrm{~m}$ vertical offset from the ADCP) collected temperature and salinity measurements to compliment the temperature measurements made by the ADCP to calculate and correct the speed of sound (which is used to calculate altimeter distance).

The four slant beams of the ADCP measured velocity profiles, while the fifth vertical beam acted as an altimeter (Figure 1) and measured the distance to the surface (either the water surface or ice bottom). The vertical beam has a beam width of $2.9^{\circ}$, so for the deployment depths here, the width of the ensonified area was roughly $2.3 \mathrm{~m}$ for SODA-A, $2.1 \mathrm{~m}$ for SODA-B, and $1.4 \mathrm{~m}$ for SODA-C. The ADCPs operated with two concurrent sampling plans: "Average + Ice", and "Burst + Waves". For both modes, the ice draft was derived from the difference between the water depth (determined by instrument pressure) and altimeter distance, after making corrections for ADCP tilt, speed of sound, and atmospheric pressure variations (e.g., Krishfield et al., 2014; Magnell et al., 2010).

During the Average + Ice sampling mode, the ADCP measured altimeter distance, water column velocity, and ice drift velocity. Ice drift velocities were measured using the instrument's built-in ice-tracking mode, which functions similarly to traditional ADCP "bottom-tracking": A ping is emitted separate to the water velocity-measuring pings with longer pulse-length that fully ensonifies the ice area for the full beam width and provides velocity measurements that are typically more accurate than in the water column (e.g., Belliveau et al., 1989). Measurements of each of the variables were provided every $10 \mathrm{~min}$ based on raw data collected in 1-min long ensembles at a sampling rate of $1 \mathrm{~Hz}$ (reported measurements are ensemble-medians after quality control processing of the raw data). The water velocities were measured in 2-m vertical range bins. Due to sidelobe interference, the upper $\sim 10 \%$ of each vertical velocity profile (2.7-4.5 m) was discarded, so near-ice logarithmic boundary layers could not be directly observed. At each time step the velocity profiles were interpolated to find the horizontal velocity, $\boldsymbol{u}_{0}$, at a fixed reference depth, $z_{\text {ref }}$; here, $z_{\text {ref }}=10 \mathrm{~m}$ to conform to the Tsamados et al. (2014) parameterization scheme. The 10-min sampled Average + Ice measurements of $\boldsymbol{u}_{i}, \boldsymbol{u}_{0}$, and $d_{i}$ were bin-averaged in 1-h bins to match the atmospheric re-analysis measurements used (see below). Figure S1 shows examples of the timeseries of each of the velocity components at SODA-B.

As indicated by its name, the Burst + Waves plan is designed for the measurement of surface gravity waves using altimeter measurements from the vertical beam. However, those altimeter measurements can also be used for measuring under-ice geometry (e.g., ice keels; Magnell et al., 2010). In Burst + Waves mode, the ADCPs measured "bursts" of data containing 2,048 samples at a rate of $2 \mathrm{~Hz}$, so each burst length was $1,024 \mathrm{~s}(\sim 17 \mathrm{~min})$. These bursts were collected once every two hours. Because the Burst + Waves and Average + Ice measurement plans were concurrent, the ADCPs recorded two values of the ice drift speed during each burst. Using the mean of those two ice drift measurements, the sampling time for each burst was converted to an along-burst distance. Within each burst, ice draft data were despiked using a moving-median outlier criteria in 127-point windows (outliers are identified as points more than three scaled median absolute deviations from the median, and replaced with linearly interpolated values). Then, the ice draft from Burst + Waves sampling were used to characterize the ice geometry (see Section 3.3).

We used atmospheric forcing from the European Center for Medium-Range Weather Forecasts (ECMWF) Reanalysis version 5 (ERA5; Hersbach et al., 2020). ERA5 provides hourly measurements at a $0.25^{\circ} \times 0.25^{\circ}$ grid resolution. A recent comparison with in situ measurements in the Eastern Arctic showed that of the six re-analysis products assessed, ERA5 provided the best representation of wind speed (which is the primary variable of interest here) during winter and spring, and second best (by a small margin) during summer (Graham et al., 2019). To generate a timeseries of atmospheric forcing at each mooring, grid points were averaged within a $30 \mathrm{~km}$ radius centered at each of the mooring locations (14-16 gridpoints per mooring). There is a degree of uncertainty in re-analysis wind measurements in the Arctic (particularly in the marginal ice zone; e.g., Brenner et al., 2020). Nonetheless, there is strong coherence between the re-analysis wind velocities and the in situ measured ice drift velocities (not shown) and associated high correlations between the two (correlation coefficients of $r=0.69,0.75$, and 0.63 for SODA-A, -B, and -C, respectively). To test sensitivity, wind velocities at the mooring locations were also found using two alternative re-analysis products: Modern-Era Retrospective analysis for Research and Applications version 2 (MERRA-2; Gelaro et al., 2017) 


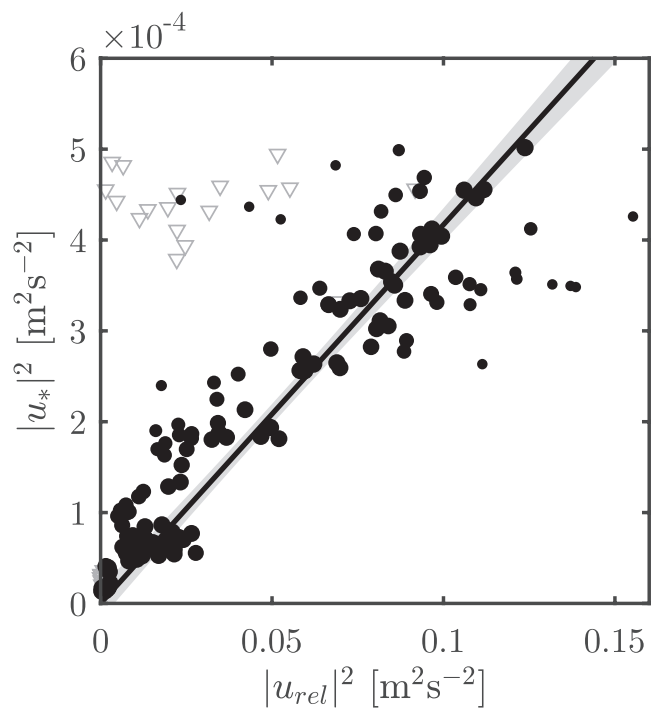

Figure 3. Example of quadratic-drag-law fit between hourly values of observed relative velocity $\left(\left|\boldsymbol{u}_{r e l}\right|^{2}=\left|\boldsymbol{u}_{i}-\boldsymbol{u}_{0}\right|^{2}\right)$, and calculated friction velocity $\left(|\boldsymbol{u} *|^{2}=\left|\tau_{i 0}\right| / \rho_{0}\right)$ from the force-balance approach (Equation 3). Black points show values used in the fitting procedure, with point sizes an indicator of the relative weighting determined by the robust fitting method. Gray triangles show points rejected from the fit by the $2 \%$-rule and demonstrate the utility of the wind factor to filter points that are not in free drift. The black line shows the regression line with $95 \%$ confidence interval shaded in gray. Data correspond to 1 week of measurements in November 2018 at SODA-A. and Japanese 55-year Reanalysis (JRA-55; Kobayashi et al., 2015, which is on a slightly coarser grid in both space and time). Across these different products, wind velocities at the mooring locations were very similar; MERRA-2 wind velocities were correlated with ERA5 winds with $r=0.97$ across all three moorings, and JRA-55 were correlated with ERA5 with $r=0.96$ (after subsetting ERA5 to the same timestamps). Resulting drag coefficient measurements (calculated per Section 3.2) were correlated with $r=0.94$ between MERRA-2 and ERA5, and $r=0.84$ between JRA-55 and ERA5. Thus, the results presented in this study are not overly sensitive to the choice of re-analysis product used.

\subsection{Application of the Force-Balance Approach}

Following McPhee (1980; see also Dewey, 2019; Randelhoff et al., 2014), we use a force-balance approach (Equation 3) to calculate the ice-ocean stress, $\tau_{i o}$. Then the ice-ocean drag coefficient, $C_{i o}$, is inferred from the quadratic drag law (Equation 4a).

The ice-ocean stress $\left(\tau_{i 0}\right)$ is calculated hourly with Equation 3 using data from the ADCP measurements and ERA5 re-analysis. The ice draft $\left(d_{i}\right)$ and ice velocity $\left(\boldsymbol{u}_{i}\right)$ are from the 1-h-averaged ADCP measurements. The local acceleration $\left(\frac{\partial \boldsymbol{u}_{i}}{\partial t}\right)$ is the numerical derivative of the 1-h-averaged $\boldsymbol{u}_{i}$ values. The geostrophic velocity $\left(\boldsymbol{u}_{g}\right)$ is estimated as the depth-averaged velocity between 5 and $20 \mathrm{~m}$ (based on results by Armitage, Bacon, et al. [2017]), and low-pass filtered with a 2-day cutoff (the result is insensitive to these choices for $\boldsymbol{u}_{g}$; see Text S1). The atmosphere-ice stress $\left(\tau_{a i}\right)$ is determined using the quadratic drag law (Equation $4 \mathrm{~b}$ ), with $10-\mathrm{m}$ wind velocity and surface air density taken from ERA5 re-analysis and $C_{a i}$ parameterized as a function of ice concentration (following ECMWF, 2019; see Text S2). In mixed ice-open water conditions, the atmosphere-ice stress, $\boldsymbol{\tau}_{a i}$, used in Equation 3 is distinct from the total atmospheric stress (Equation 5b). Because Equation 3 assumes that ice is in free drift, values for which the wind factor $\left(\left|\boldsymbol{u}_{i}\right| /\left|\boldsymbol{u}_{a}\right|\right.$; determined hourly) was less than $2 \%$ were rejected (the so-called "2\%-rule"). The use of wind factor as a filtering criteria implies an intermittency of internal ice stresses, which is consistent with Steele, Zhang, et al. (1997), who found that on short timescales the atmospheric stress input to the ice $\left(\tau_{a i}\right)$ was primarily balanced by only one of either the ocean-ice stress $\left(\tau_{o i}\right)$ or the internal ice stress. $(\nabla \cdot \sigma)$. The friction velocity $\left(\boldsymbol{u}_{*}\right)$ is determined from $\tau_{i o}$ assuming a constant $\rho_{o}=1,025 \mathrm{~kg} \mathrm{~m}^{-3}$ (with the definition $\tau_{i o}=\rho_{o} \boldsymbol{u}_{*}\left|\boldsymbol{u}_{*}\right|$ ).

To calculate the ice-ocean drag coefficient, the record is split into windows. Within each window the quadratic drag law (Equation 4a) is applied by regressing hourly calculated values of $\left|\boldsymbol{u}_{*}\right|^{2}$ (as described above) with hourly measured $\left|\boldsymbol{u}_{r e l}\right|^{2}$ (with $\boldsymbol{u}_{0}$ defined at a 10-m reference depth). Then the value of $C_{i o}$ is the slope of the regression line (Figure 3). Windows are chosen to be 7 days in length, which provides an average of 80 points in each window (after using the $2 \%$-rule to exclude non-free-drift points). Based on average ice drift speeds, each window covers roughly $75 \mathrm{~km}$ of ice (though there is both spatial and temporal variability in the actual window size). While shorter window lengths can resolve some higher frequency variability at the expense of larger uncertainties, the overall seasonal patterns found here are not sensitive to the window length chosen. Regression is performed with a bisquare robust linear fitting algorithm and forced through the origin (Huber, 1981). This method iteratively reduces the weighting on outliers, which may occur, for example, from intermittent violation of the free-drift assumption. Performing regression within windows instead of calculating $C_{i o}$ on a point-by-point basis (as in Dewey, 2019) minimizes the effects of noise and uncertainty (particularly for low values of $\boldsymbol{u}_{r e l}$ ), which may have resulted from a combination of measurement noise, higher frequency temporal variations, or unaccounted stresses (e.g., internal ice stress). Calculated values of the drag coefficient were rejected if the uncertainty in $C_{i o}$ was $\geq 2.5 \times 10^{-3}$ (based on a $t$-test with 95\% confidence interval; Bendat \& Piersol, 1971). High uncertainties in $C_{i o}$ occurred most frequently in winter when many of the data were rejected due to free drift conditions not being met. Tests 

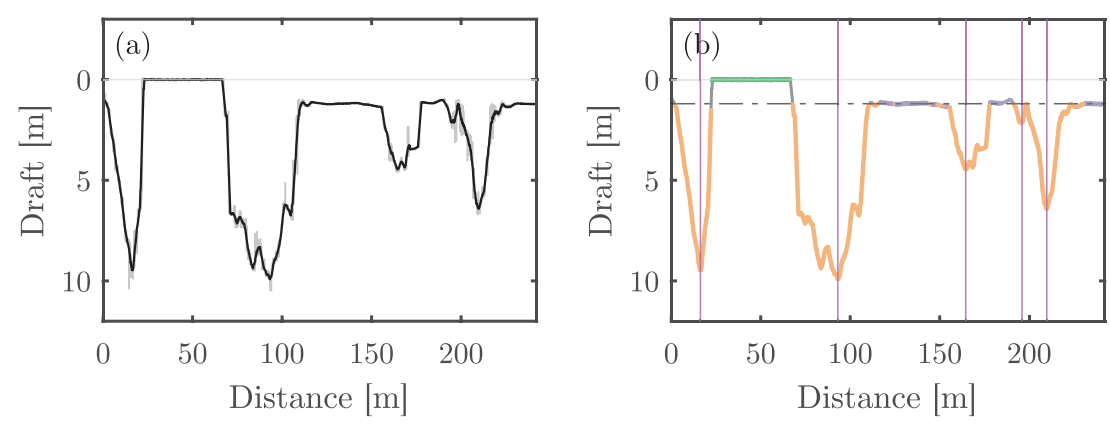

Figure 4. Example of ice draft from burst measurements: (a) Raw (thin gray line) and smoothed (black line) ice draft during a single burst ( 17 min) in April 2019 at SODA-A. (b) The burst from (a) classified to show leads (green line), level ice (purple), and ridged ice (orange), with vertical magenta lines showing unique keels (based on Rayleigh criterion), and black dashed-dotted line showing the level ice draft classified for that burst.

using non-linear fits of the form $\left|\tau_{i 0}\right| \propto\left|\boldsymbol{u}_{\text {rel }}\right|^{n}$ (see Section 5.1) did not produce better fits than the quadratic drag law with $n=2\left(r^{2}\right.$ values from $n \neq 2$ fits were approximately equal to those with $\left.n=2\right)$. Given the direct concurrent and collocated measurements of the ice and ocean velocities here, it is not necessary to exclude periods of small ice-ocean relative velocity, a condition often necessary when using satellite remote sensing to estimate ocean velocities (e.g., in McPhee, 1980).

This method of drag calculation essentially asks what value of $C_{i o}$ would be required to reproduce the observed sea ice motion. In doing so, the method effectively integrates over both the temporal intermittency and the spatial heterogeneity of turbulent momentum fluxes across ice floes and thus provides bulk-average drag coefficient values. These resulting drag coefficients are appropriate for comparison to model parameterizations as the goal of those parameterizations is to provide a bulk coefficient for use within a model grid cell.

There is no physical basis to expect that the relationship between total ocean stress, $\tau_{o c n}$, and wind speed should follow the quadratic drag law, so the linear fitting procedure used to calculate $C_{i 0}$ cannot be similarly applied to find $C_{\text {equiv }}$. Instead, $C_{\text {equiv }}$ is computed on a point-by-point (hourly) basis using Equation 7, with $\tau_{\text {ocn }}$ given by Equation $5 \mathrm{a}$ and with $A$ from ERA5. For points defined as being in free-drift (based on the $2 \%$ rule), the ice-ocean stress, $\tau_{i 0}$ used in Equation 5 a is the same as described above (Equation 3 ). The analysis was extended beyond free-drift periods by calculating $\tau_{i o}$ for those times using Equation $4 \mathrm{a}$ and values of $C_{i o}$ from the regression procedure, interpolated to points with a wind factor $<2 \%$.

\subsection{Ice Geometry}

During periods of ice cover, the ADCP Burst + Waves sampling provides one dimensional (along-drift) tracking of the under-ice geometry (Figure 4a). We use these to quantify the geometric characteristics used in the parameterization schemes in Section 2. Importantly, the fixed mooring platforms allow for sampling across a broad range of different ice conditions as they evolve over the annual cycle.

Spectral analysis is used as part of a filtering criteria to separate ice-covered conditions from open water conditions; this ensures that surface gravity waves are not erroneously misidentified as ice keels. For each burst, frequency spectra of measured altimeter distances are constructed. Surface gravity waves have distinct and well known spectral shapes (e.g., Phillips, 1985), with peaks at relatively high frequencies ( $\gtrsim 0.04 \mathrm{~Hz})$, while sea ice has broadly distributed spectral energy with energy concentrated at lower horizontal wavenumbers (which translate to low frequencies) (e.g., McPhee \& Kantha, 1989). Following Shcherbina et al. (2016) and Kirillov et al. (2020), ice-covered conditions are identified using the ratio of integrated spectral energy in low- and high-frequency bands (using a cutoff frequency of $0.1 \mathrm{~Hz}$, based on observed conditions): burst are deemed to be ice-covered when the ratio of high-to-low frequency spectral energy is less than 5 . Then, bursts identified as being in open-water but with measured non-zero level ice draft, $d_{l v l}$, provide a secondary empirical correction to ice draft measurements to account for water-column sound-speed variations (e.g., 
due to shallow stratification; Kirillov et al., 2020). These corrections were small, and primarily applied to marginal ice covered periods.

For each ice-covered burst we quantify the draft of level ice, the extent and number of leads, and the number and size of keels (Figure 4b). Prior to classification, bursts are smoothed with a moving-average filter using a centered window with a width of $2 \mathrm{~m}$ (because of variability in ice drift speed, the number of points in each window varies from burst to burst). Bursts frequently contained apparent leads, identified as all points in a burst with a measured draft below a tolerance level (taken as $0.15 \mathrm{~m}$ to account for instrument noise and uncertainty associated with both atmospheric pressure variations and sound speed). Strictly, this procedure is unable to differentiate between open-water leads and refrozen leads containing thin ice, but from the perspective of the drag parameterizations (Section 2), both scenarios are dynamically equivalent in that they both contribute to the floe edge form drag. Within each burst, level ice is defined by a local gradient less than 0.025 (equivalent to the process in Wadhams \& Horne, 1980) and a draft of less than $3 \mathrm{~m}$ (roughly the limit of thermodynamic growth; Maykut \& Untersteiner, 1971). The level ice draft for each burst is then taken as the median draft of all ice identified as level within the burst. In cases where no level ice was identified (i.e., the entire burst measured ridged ice), the level ice draft is found by interpolating across adjacent bursts. Keels identification follows Martin (2007), using a Rayleigh criterion to define unique keels (see also Wadhams \& Davy, 1986; Wadhams \& Horne, 1980; Williams et al., 1975) with a minimum keel depth cutoff of $0.5 \mathrm{~m}$ relative to the level ice draft for that burst. Relative keel depths at each of the moorings closely followed exponential probability distributions (not shown), which is in line with previous literature (e.g., Wadhams \& Davy, 1986; Wadhams \& Horne, 1980), and a total of 14,694 individual keels are identified throughout the full study period (6,282, 4,305, and 4,107 at SODA-A, -B, and -C, respectively). The maximum relative keel depth measured at any of the moorings through the full deployment was $11.4 \mathrm{~m}$ at SODA-B. Keel sizes across the three moorings were fairly similar.

The parameterized ice-ocean drag is based on statistical descriptions of the ice geometry (see Section 2). Statistics are accumulated over one-week periods to be consistent with the windowing procedure for the ice-ocean drag (Section 3.2). The keel depth $\left(h_{k}\right)$ and level ice draft $\left(d_{l v l}\right)$ are simply averages of individual measurements taken for all bursts in each window. The average keel spacing $\left(\ell_{k}\right)$ is taken as the total distance measured by all bursts in a given window (both ice and open water) divided by the total number of keels counted during that window. Except for some bursts in the marginal ice zone, floe lengths are typically longer than the distance measured by an individual burst. To estimate an average floe length $\left(\ell_{f}\right)$ the total measured ice-covered distance for a given window is divided by the number of leads counted in that window. Similarly, the average lead length $\left(\ell_{l}\right)$ was the total open water distance divided by the number of leads. These definitions for $\ell_{k}$ and $\ell_{f}$ are consistent with their inclusion in parameterizations (Lu et al., 2011; Tsamados et al., 2014). A local average daily ice concentration $(A)$, was also calculated using burst data as a ratio of the total measured ice-covered distance to the total distance measured by all bursts (ice and open water). Using $A$, the average lead length can be written as $\ell_{l}=\ell_{f}(1-A) / A$ for one-dimensional measurements (Lu et al., 2011). The values $\ell_{f}$ and $\ell_{l}$ are only defined for ice concentration less than $100 \%$. The measurements show seasonal signals in all of the measured geometry statistics at all moorings (Figure 5). Despite both $d_{l v l}$ and $\ell_{f}$ decreasing in the summer/fall (Figures $5 \mathrm{a}$ and $5 \mathrm{c}$ ), the much wider range of variation of $\ell_{f}$ (over roughly three order of magnitude) compared to $d_{l v l}$ results in floe aspect ratios $\left(d_{l v l} /\right.$ $\ell_{f}$ ) that are elevated in the fall (Figure 5e). The relative keel depths and spacing $\left(h_{k \text { rel }}\right.$ and $\left.\ell_{k}\right)$ appear to have some negative correlation (cf., Figures $5 \mathrm{~b}$ and $5 \mathrm{~d}$ ), so that both signals contribute to the minimum ridging intensity $\left(h_{k} / \ell_{k}\right)$ in the summer/fall (Figure $\left.5 \mathrm{f}\right)$.

\subsection{Implementing Model Parameterization Schemes}

Four different variations of ice-ocean drag parameterizations were tested. These are summarized in Table 2. In the first two variations (labeled L11 and T14(I), respectively), direct measurements of the sea ice geometry (Section 3.3) were used to test the parameterization schemes proposed by Lu et al. (2011) and Tsamados et al. (2014) (Section 2.1) using default parameter values in each scheme. We introduce an alternative version of the Tsamados et al. (2014) scheme, labeled T14(II), which uses slightly modified geometry definitions and coefficient values, and still uses direct ice geometry measurements. Finally, the T14(III) variation 

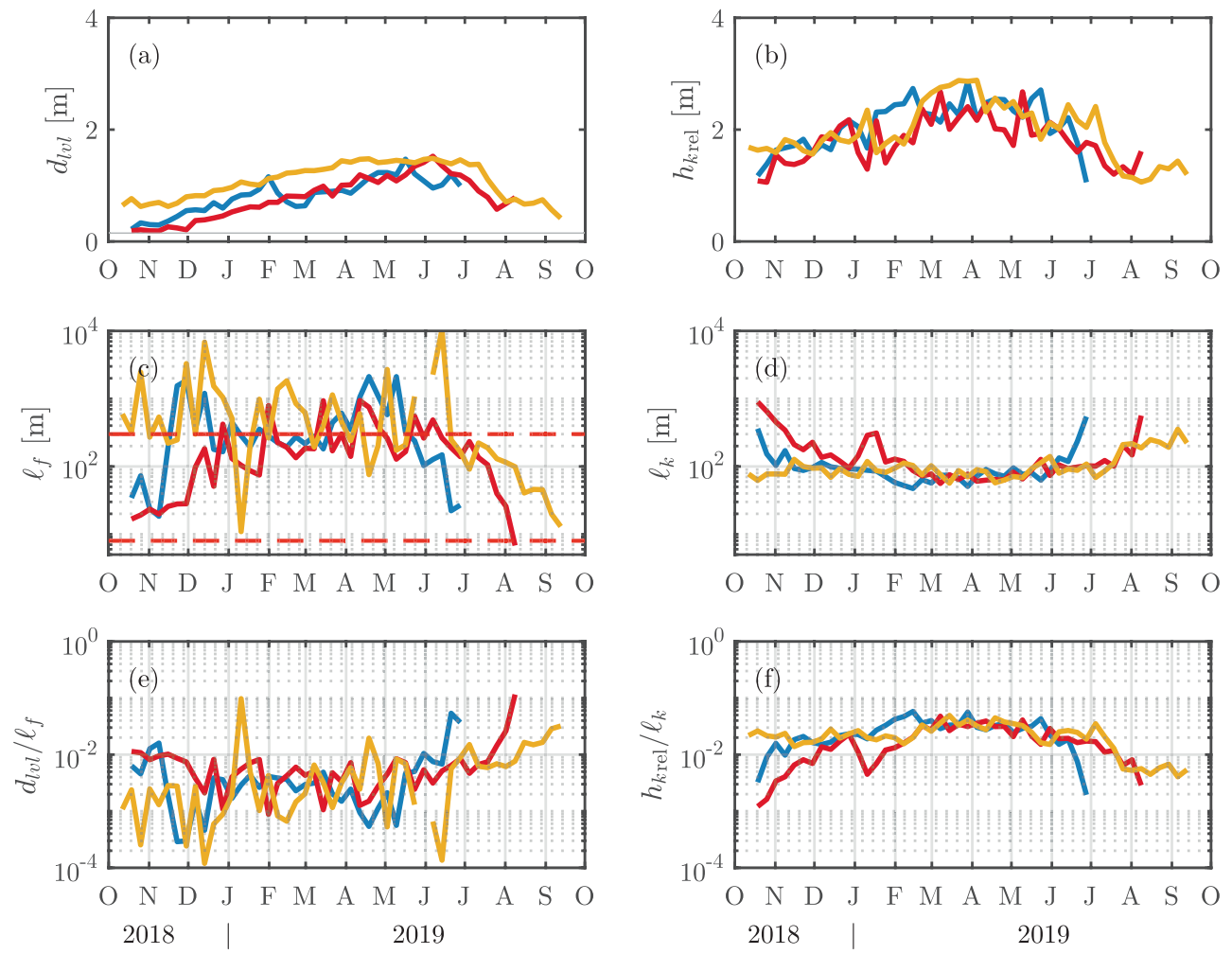

SODA-A SODA-B SODA-C

Figure 5. Weekly statistics of sea ice geometry for each mooring: (a) mean level ice draft; (b) mean relative keel depth; (c) mean floe length; (d) mean keel spacing (e) aspect ratio $\left(d_{l v} / \ell_{f}\right)$; and (f) ridging intensity $\left(h_{k} / \ell_{k}\right)$. Horizontal dashed red lines in (c) show the maximum and minimum extents of the parameterized floe length (Equation 14).

Table 2

Summary of Parameters and Functions Used in the Parameterization Schemes Tested

\begin{tabular}{lcccc}
\hline & L11 & T14(I) & T14(II) & T14(III) \\
\hline$c_{f}$ & 1 & 1 & $0.3^{\mathrm{a}}$ & 1 \\
$c_{k}$ & $1 / \pi$ & 0.2 & $0.4^{\mathrm{a}}$ & 0.2 \\
$c_{s}$ & $2 \times 10^{-3}$ & $2 \times 10^{-3}$ & ${\text { Equation } 12^{\mathrm{b}}}^{-4}$ & $2 \times 10^{-3}$ \\
$z_{0 i}$ & $\mathrm{n} / \mathrm{a}$ & $5 \times 10^{-4} \mathrm{~m}$ & $1 \times 10^{-3} \mathrm{~m}$ & $5 \times 10^{-4} \mathrm{~m}$ \\
$z_{0 w}$ & $\mathrm{n} / \mathrm{a}$ & $3.27 \times 10^{-4} \mathrm{~m}$ & $3.27 \times 10^{-4} \mathrm{~m}$ & $3.27 \times 10^{-4} \mathrm{~m}$ \\
$m_{w}$ & 10 & 10 & 10 & 10 \\
$s_{l}$ & $\mathrm{n} / \mathrm{a}$ & 0.18 & 0.18 & 0.18 \\
$S_{c}$ & Equation $10 \mathrm{~b}$ & Equation $10 \mathrm{a}$ & Equation $10 \mathrm{a}$ & Equation 10a \\
$P_{0}$ & $\mathrm{n} / \mathrm{a}$ & Equation 11 & Equation $11^{\mathrm{b}}$ & Equation 11 \\
$h_{k}$ & meas. $h_{k \text { rel }}$ & meas. $h_{k \text { tot }}$ & meas. $h_{k \text { rel }}$ & Equation 13a \\
$\ell_{k}$ & meas. & meas. & meas. & Equation 13b \\
$\ell_{f}$ & meas. & meas. & meas. & Equation 14 \\
\hline
\end{tabular}

Abbreviation: n/a, not applicable; meas., measured (see Section 3.3). aparameters adjusted based on best fit to observations in this study. ${ }^{\mathrm{b}}$ using a relative reference depth $\left(z_{\text {ref }}-d_{l v l}\right)$ tested a combination of both physics and ice geometry parameterization from Tsamados et al. (2014), and thus is most comparable to modeling efforts where geometry measurements are not available.

The T14(II) scheme is a modification of the T14(I) scheme, introduced for this study. It still uses the direct measurements of sea ice geometry, but uses the relative definitions of keel depth and reference depth (see Section 2.1). Additionally, in T14(II), some of the parameters have been changed from their default values. The skin drag coefficient of resistance $\left(c_{s}\right)$ is replaced with Equation 12 and the roughness length associated with level ice bottom, $z_{0 i}$ is replaced with a value of $1 \times 10^{-3} \mathrm{~m}$, which is reflective of observations of ice with no significant morphology (McPhee, 2002; McPhee et al., 1999). With this $z_{0 i}$ and a 10-m reference depth, the value of $c_{s}$ calculated for a $1-\mathrm{m}$ ice draft is $2 \times 10^{-3}$, which is the same as in T14(I); however, the use of Equation 12 allows $c_{s}$ to vary slightly through the year as the ice draft changes seasonally, and gives it an explicit dependence on $z_{\text {ref }}$. By using this formulation $c_{S}$ is no longer a free parameter. Finally, the coefficients of resistance $c_{f}$ and $c_{k}$ have been replaced with values that provide the closest fit between parameterized and observed drag coefficient values when considered across all moorings. These values were found with multiple linear regression: first the values $c_{f}$ and $c_{k}$ in Equations $9 \mathrm{a}$ and $9 \mathrm{~b}$ were set to 1 , then resulting $C_{f}$ and $C_{k}$ from all moorings were regressed against the residual observed drag after subtracting the skin drag component, $C_{i o}-C_{s}$; the regression coefficients 
then gave the new values of $c_{f}$ and $c_{k}$ which were used in T14(II). While used as fitting parameters here, $c_{f}$ and $c_{k}$ should be reflective of the individual geometries of the floe edges and keels. For example, the value of $c_{k}=0.4$ found with this method corresponds to a keel slope angle of $19.6^{\circ}$ based on the fit to experimental results by $\mathrm{Zu}$ et al. (2020) (noting that their definition of $C_{k}$ introduces a factor of $\pi / 2$ difference in values of $c_{k}$ compared to this study), which is close to the mean keel slope of first year ridges of $\alpha_{k}=26.6^{\circ}$ found by Timco and Burden (1997), and the value of $\alpha_{k}=22^{\circ}$ used in the parameterization by Tsamados et al. (2014). Note that the T14(II) scheme does not reflect a full optimization tuning of all of the available parameters, nor is it a rigorous fitting approach for $c_{f}, c_{k}$ (as discussed in Section 5.2).

As the ADCP measurements provide direct observations of ice geometry (Section 3.3), the parameterization of ice geometry (Section 2.2) is not necessary in order to implement Equation 9 in L11, T14(I), and T14(II). Instead, this allows us to separately test the physics parameterization (Section 2.1) and the geometry parameterization (Section 2.2). To do so, a final variation (T14(III)) is tested that uses the default parameter values from Tsamados et al. (2014) but instead of using the direct measurements of sea ice geometry, geometry statistics are estimated using bulk measurements and Equations 13 and 14.

Application of Equation 13 using ADCP measurements provides some challenges. The ice volume $\left(v_{r d g}\right)$ and areas $\left(a_{r d g}, a_{i}\right)$ in Equation 13 are fundamentally defined over a two dimensional area (i.e., within a model gridcell), but the ADCP draft measurements are one dimensional (along-drift). To adapt our measurements to apply Equation 13, we calculate $v_{r d g}, a_{r d g}$, and $a_{i}$ on a per-unit-width basis. However, the relative angles between the keel orientations and the direction of sampling (which is unknown) will cause an overestimate of the area or volume of the feature unless measurements are made perpendicular to the keels. Fortunately, this mismatch creates an equal bias for both volume and area calculations, so the ratio $v_{r d g} /$ $a_{r d g}$ in Equation 13a is not impacted. However, due to crossing angle mismatch, extra care must be taken when calculating and interpreting $\ell_{k}$ from Equation 13b. If both keels and leads are linear features whose orientations follow the same statistical distributions, then the ratio $a_{i} / a_{r d g}$ measured with along-drift data will approximate the true (two-dimensional) value if averaged over a sufficiently large sample of keels and leads. However, in full ice cover leads are relatively scarce while in the marginal ice zone it may not be appropriate to consider leads to be linear features. It is unclear whether one-dimensional sampling of $a_{i}$ will introduce any mean bias. For a uniformly distributed keel orientation, one-dimensional sampling will lead to a mean over-estimate of $a_{r d g}$ by a factor of $\pi / 2$. On that basis $a_{r d g}$ are multiplied by a $2 / \pi$ correction factor when applying Equation 13b.

\section{Results}

\subsection{Seasonal and Spatial Variation of Ice-Ocean Drag}

For all three moorings, the force-balance approach provided estimates for the ice-ocean drag coefficient, $C_{i o}$, throughout the full annual cycle (Figure 6) even despite some winter data gaps (due to higher frequencies of internal stresses). These estimated values of the ice-ocean drag coefficient exhibit both spatial and seasonal variations.

Drag coefficients measured at SODA-A and SODA-B (the two southern moorings; Figure 2a) show a similar seasonal behavior. For both, the drag coefficients start at low values $\left(C_{i o} \sim 2-3 \times 10^{-3}\right)$, and steadily increase through the winter to a maximum in spring (April to May) before declining (Figures $6 \mathrm{~b}$ and $6 \mathrm{c}$ ). The decrease of $C_{i o}$ is more gradual at SODA-B than SODA-A, and summertime minimum values at SODA-A are lower than at SODA-B (cf., Figures $6 \mathrm{~b}$ and $6 \mathrm{c}$ ). The timing of the shift from increasing to decreasing $C_{i o}$ at these two moorings is roughly coincident with the change from net surface cooling to net surface heating in the atmospheric re-analysis data, which occurred in April to May.

In contrast, the record at SODA-C begins with an elevated drag coefficient $\left(C_{i o} \sim 6 \times 10^{-3}\right)$ which remains roughly constant from fall through spring (Figure $6 \mathrm{a}$ ). After the shift to net atmospheric surface heating in Apr.-May, there may be a slight decline in $C_{i o}$, but values are still elevated for some months, until there is a sharp drop in early to mid-July. This sudden drop in ice-ocean drag is associated with a similar sharp decline in both floe sizes (Figure 5c) and ridging intensity (Figure 5f), suggesting a dramatic ice breakup and melting event occurred. 

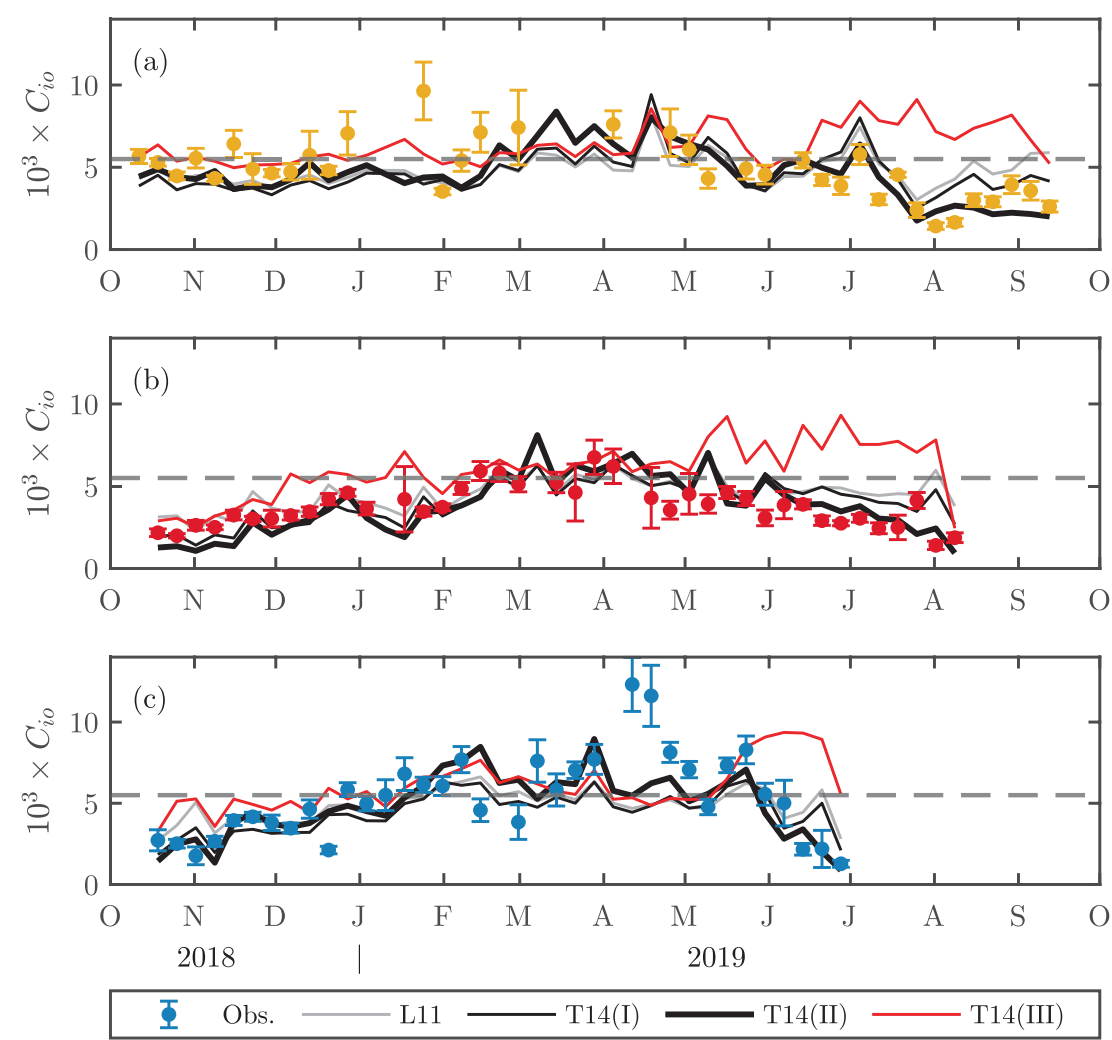

Figure 6. Timeseries of ice-ocean drag coefficients from north-to-south: (a) SODA-C, (b) SODA-B, and (c) SODA-A. In each panel, points with error-bars (colored by moorings per Figure 2a) show the values of $C_{i o}$ calculated with the force-balance approach (labeled "Obs".), while lines correspond to the different variations of parameterization schemes (Table 2), as indicated by the legend. Error bars show 95\%-confidence interval bounds from the linear fitting procedure. The horizontal gray dashed line shows the value of $C_{i o}=5.5 \times 10^{-3}$ for comparison.

At all three moorings, drag coefficient values from mid-winter to spring are similar to each other, and fluctuate near or above the canonical value of $C_{i o}=5.5 \times 10^{-3}$. However, differences between the moorings in fall and summer imply large-scale spatial gradients in the ice-ocean drag coefficient across the Beaufort Sea. Section 4.3 discusses morphological drivers of the observed seasonality in greater depth.

\subsection{Evaluation of Parameterization Schemes}

Ice-ocean drag coefficients calculated with all of the tested parameterization schemes (Table 2) show values and temporal variability that broadly match the values observed with the force-balance approach (Figure 6). This agreement indicates that variability of ice-ocean drag can be primarily explained by seasonal changes in the ice morphology and the associated skin/form drag contributions. Despite general success, some versions of the parameterization schemes are better performing. In particular, while the T14(III) scheme provides a reasonable match at all moorings in the early part of the record, it diverges significantly from the observations in the latter half of the record, and even reaches a maximum $C_{i o}$ in summer/fall when the observations show a minimum. Figure 7 shows direct comparisons of the observed and parameterized values for each of the four test schemes. There is good agreement between the observed drag coefficients and those predicted by both L11 and T14(I) when $C_{i o}$ are low $\left(\lesssim 5 \times 10^{-3}\right)$; for higher values of $C_{i o}\left(\gtrsim 5 \times 10^{-3}\right)$, there is a roll-off of the modeled values (Figures $7 \mathrm{a}$ and $7 \mathrm{~b}$ ). Values from T14(II) follow the one-to-one line across the full range of $C_{i o}$ (Figure 7c), while those from T14(III) are mostly above the one-to-one line and do not present any recognizable correlation with force-balance observations. A few notable outliers exist that are not described by any of the model schemes (e.g., high observed values of drag in mid-April at SODA-A; Figure 6a), potentially suggesting other sources of drag (e.g., internal wave drag) that cannot be explained by ice geometry variations alone; however, these points are fairly limited. 
L11

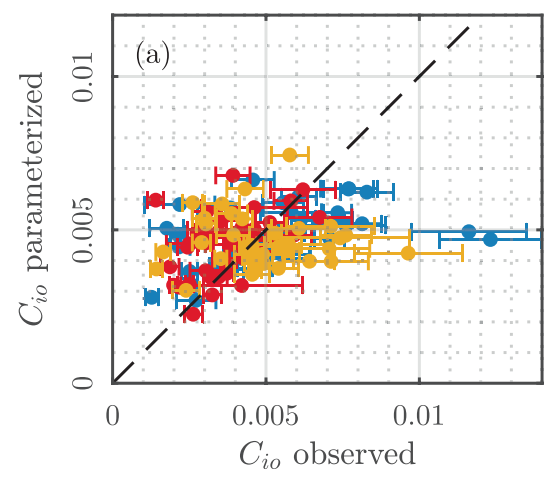

T14(II)

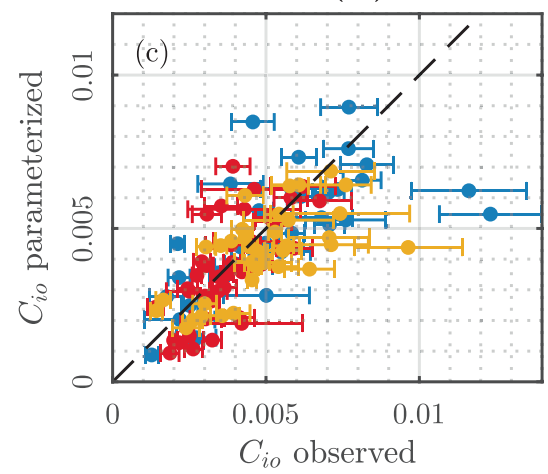

$\mathrm{T} 14(\mathrm{I})$

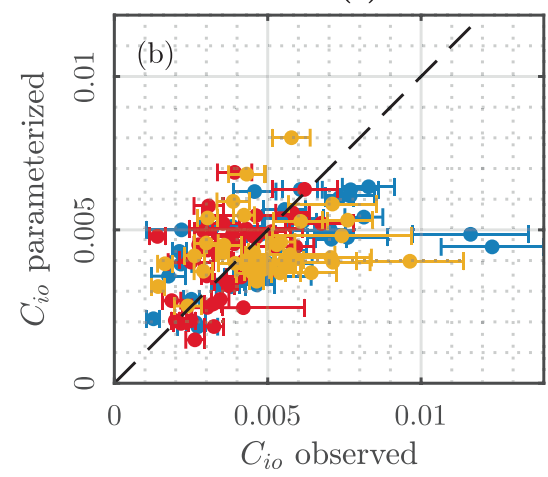

T14(III)

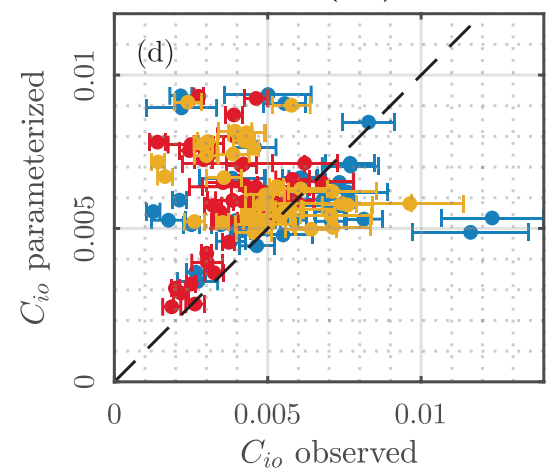

\section{$\mapsto$ SODA-A $\mapsto-1$ SODA-B $\mapsto-1$ SODA-C}

Figure 7. A comparison between the ice-ocean drag coefficients determined using the force-balance approach ("observed"), and using the different variations of geometry-based parameterization: (a) L11, (b) T14(I), (c) T14(II), and (d) T14(III). In each panel, the black dashed line shows the one-to-one slope, and the points are colored by mooring according the legend.

These statements are corroborated by quantitative assessments of model performance across all moorings (Table 3). Values from both L11 and T14(I) have weak correlations with observations $\left(r^{2}=0.13\right.$ and 0.22 , respectively). T14(I) has a slightly negative normalized bias (NBI; -012), while L11 is approximately unbiased. The T14(II) scheme has the best correlation of the four tests $\left(r^{2}=0.46\right)$ and the lowest normalized root-mean-squared error (NRMSE; 0.31), though it also has a slightly negative normalized bias $(-0.09)$. When considered over the full year, the T14(III) scheme is biased high (NBI of 0.31), has high NRMSE

Table 3

Summary of Fit Statistics of Ice-Ocean Drag Coefficients Determined Using the Force-Balance Approach and Using the Different Variations of Geometry-Based Parameterization

\begin{tabular}{lccr}
\hline Scheme & $r^{2}$ & NRMSE & NBI \\
\hline L11 & 0.13 & 0.37 & -0.00 \\
T14(I) & 0.22 & 0.36 & -0.08 \\
T14(II) & 0.46 & 0.31 & -0.09 \\
T14(III) & 0.00 & 0.57 & 0.31 \\
\hline
\end{tabular}

Abbreviation: NRSME, normalized root mean square error; NBI, normalized bias.
(0.57), and is uncorrelated with observations; however, if only the early part of the record (before May 2019) is considered, the fit is better $\left(r^{2}=0.17\right.$, NRMSE $\left.=0.35\right)$. Tests in which the observed drag coefficients and geometry statistics were determined using different window lengths (ranging between 1 and $14 \mathrm{~d}$ ) all produce similar correlations as the 7-d windows presented (not shown), giving confidence that the parameterization schemes are appropriate over a wide range of scales.

\subsection{Partitioning of Drag Components and Predictions of Ice Geometry}

Parameterized ice-ocean drag coefficients are built up from three components: form drag on floe edges (Equation 9a), form drag on keels (Equation 9b), and skin drag (Equation 9c). Insofar as the ice-ocean drag 

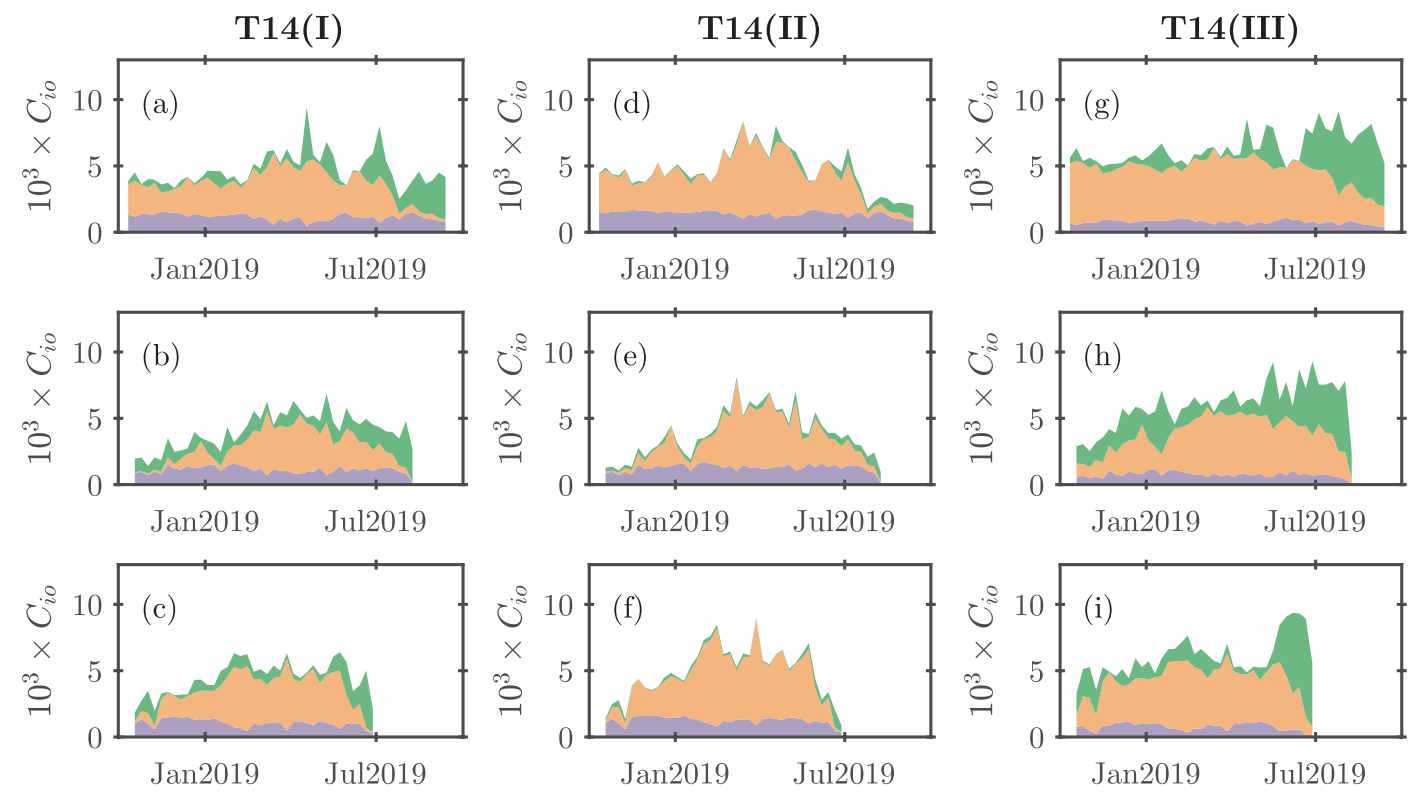

\begin{tabular}{|l|l|}
$C_{f}$ & $C_{k}$ \\
\hline
\end{tabular}

Figure 8. Stacked contributions to the ice-ocean drag coefficient $C_{i o}$ from form drag on floe edges $\left(C_{f}\right)$, form drag on keels $\left(C_{k}\right)$, and skin $\operatorname{drag}\left(C_{s}\right)$ calculated using $(\mathrm{a}-\mathrm{c})$ the T14(I) scheme, (d-f) the T14(II) scheme, and (g-i) the T14(III) scheme (see Table 2) for (a, d, and g) SODA-C, (b, e, and h) SODA-B, and (c, f, and i) SODA-A.

coefficient is driven by ice morphology, examination of the partitioning of drag components allows us to better understand the impact of those morphological variations. In all four of the parameterization schemes tested, the ice-ocean drag coefficient in the winter is largely driven by form drag on ice keels $\left(C_{k}\right)$. Skin drag $\left(C_{s}\right)$ is generally much smaller, and does not show significant seasonal variation, and floe edge drag $\left(C_{f}\right)$ becomes more important in the summer as the ice begins to melt and break apart into smaller floes. This general pattern qualitatively matches results from sea ice models (Martin, Tsamados, et al., 2016; Tsamados et al., 2014), but details vary from those model results.

For the three schemes that use direct measurements of the geometry (L11, T14(I), and T14(II)), the seasonality of $C_{i o}$ observed in Figure 6 is driven by seasonal growth and melt of ice keels, as seen by variation in $C_{k}$ (T14(I) and T14(II) are shown in Figures 8a-8f; L11 is very similar to T14(I) so it is not shown). The exact partition between $C_{f}$ and $C_{k}$ in these schemes depends on the values of the coefficients of resistance $c_{f}$ and $c_{k}$ (see Table 2), but the overall behavior is similar for the different schemes (cf., Figures $8 \mathrm{a}-8 \mathrm{c}$ and $8 \mathrm{~d}-8 \mathrm{f}$ ). At the southern moorings (SODA-A, -B), which start the timeseries in open water, there is initially only small contribution from $C_{k}$ and most of the drag is due to a combination of $C_{f}$ and $C_{s}$. As the number and size of keels grow through the year (Figure 5), so too does the contribution from $C_{k}$ (Figures $8 \mathrm{~b}, 8 \mathrm{c}, 8 \mathrm{e}$, and $8 \mathrm{f}$ ). At SODA-C, the timeseries begins in ice cover with established ridging, and $C_{k}$ is the main component of $C_{i o}$ from the onset (Figures 8a and 8d). All three moorings have some small contributions to floe edge drag throughout the full year due to the presence of (potentially refrozen) leads. Following the onset of melting conditions, an increase in floe edge drag accompanies the decline of keel drag at all locations; however, the increased floe edge drag is not enough to compensate for the lack of keels at any of the moorings (Figures 8a-8f). This contrasts the modeling results from Tsamados et al. (2014) and Martin, Tsamados, et al. (2016), which show that floe edge drag is substantial during summer/fall. While not the main focus here, it is also noteworthy that keel decline varied between the three moorings: At both the southernmost mooring (SODA-A) and northernmost mooring (SODA-C), there was a fairly rapid drop in $C_{k}$ over the period of $\sim 2$ weeks in late June and early July, respectively, due to both decreased size and number of keels (Figures $5 \mathrm{~b}$ and $5 \mathrm{~d}$ ); at SODA-B, the decrease in $C_{k}$ was more gradual. Note that at SODA-A and -B, where there was a strong seasonality in keel drag, growth of $C_{k}$ proceeded at a much slower rate than ice cover growth; at both moorings, ice concentration was close to $100 \%$ by early November (Figures $2 \mathrm{c}-2 \mathrm{e}$ ), while $C_{k}$ 

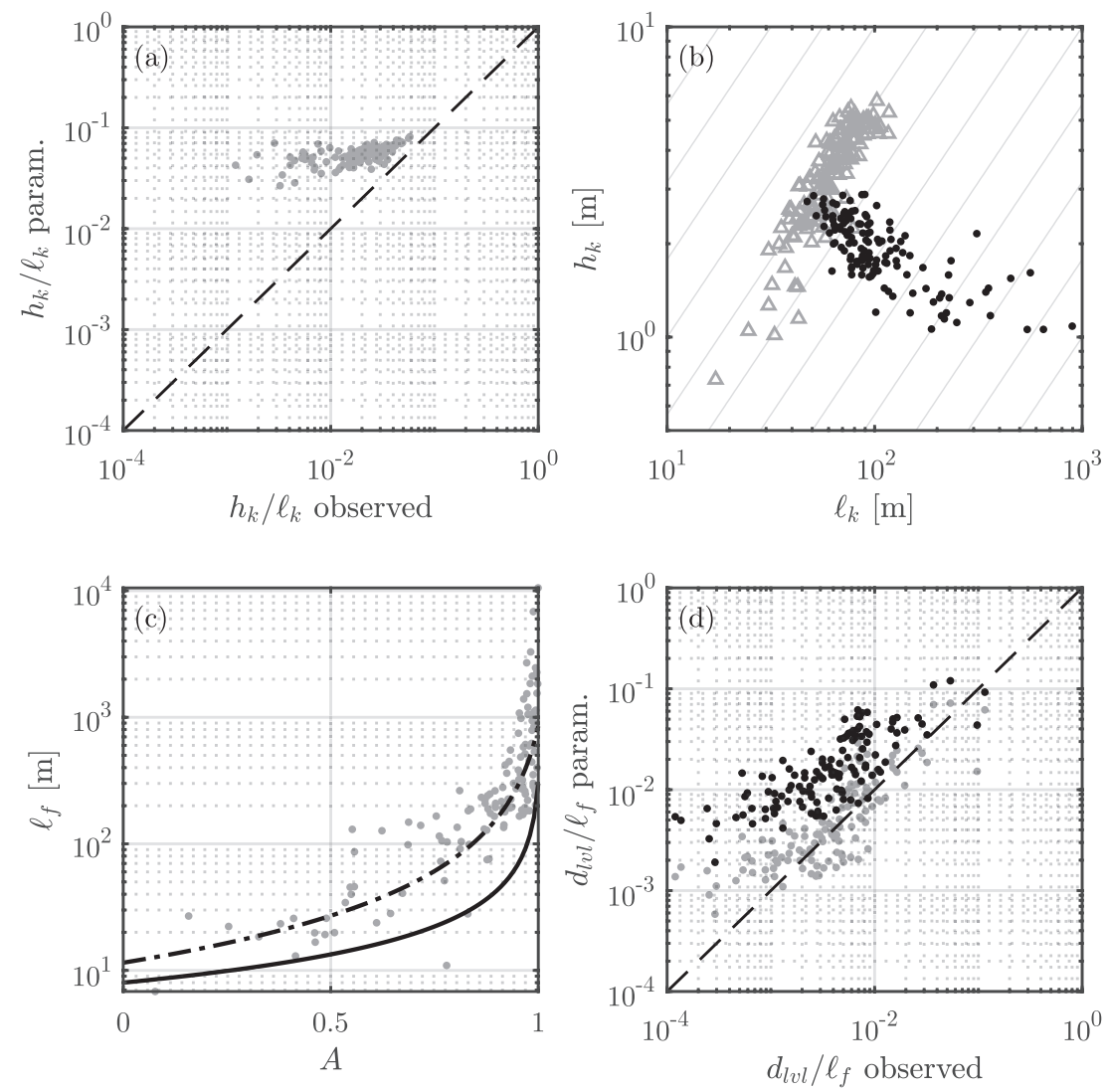

Figure 9. A comparison of observed and parameterized sea ice geometry statistics: (a) Observed versus parameterized ridging intensity $\left(h_{k} / \ell_{k}\right)$ with weekly values measured at all moorings; the black dashed line shows the one-toone slope. (b) Weekly values of ridge spacing $\left(\ell_{k}\right)$ versus keep depth $\left(h_{k}\right)$ from observations (black points) and parameterizations (gray triangles). Gray contours correspond to lines of constant $h_{k} / \ell_{k}$. Observed values of $h_{k}$ in (a and b) are relative keel depth $\left(h_{k \text { rel }}\right)$. (c) Observed floe length $\ell_{f}$ as a function of ice concentration $A$ (gray points) showing the fit of Equation 14 when using the default parameter set (solid black line), and with modified set of parameters (dashed-dotted line). (d) As per (a) but for aspect ratio $\left(d_{l v l} / \ell_{f}\right)$; black points show the aspect ratio when $\ell_{f}$ is calculated using the default parameters in Equation 14, and gray points show the aspect ratio when a modified set of parameters are used in Equation 14.

remained relatively low through January. As such, it is unlikely that ice concentration based drag parameterizations (such as are suggested for atmospheric drag; e.g., Andreas, Horst, et al., 2010a) would ever be able to sufficiently capture observed seasonal variations in $C_{i o}$.

The drag partition from the T14(III) scheme (Figures 8g-8i) differs from the results of the T14(II) scheme. While keel drag $\left(C_{k}\right)$ is still the dominant contribution during winter, its seasonality is somewhat muted compared to T14(II) (compare $C_{k}$ in Figures 8d-8f with Figures 8g-8i). More striking are the differences in floe edge drag: $C_{f}$ is much higher in the T14(III) scheme at all moorings and times of the year, and in summer/fall the increase in $C_{f}$ outpaces the associated decrease in $C_{k}$. As a result, the T14(III) scheme has the largest value of $C_{i o}$ in summer/fall, which conforms to previous model results (Martin, Tsamados, et al., 2016; Tsamados et al., 2014). While these differences can be partly attributed to the differences in coefficients of resistance between the two schemes ( $c_{f}$ and $c_{k}$, see Table 2 ), the main difference arises from the fact that the T14(III) scheme does not use direct measurements of the sea ice geometry, and instead relies on parameterized geometry statistics (Section 2.2). In the early part of the record, before $C_{f}$ becomes large, the T14(III) scheme is comparable to the other parameterization schemes.

Differences in $C_{f}$ between T14(II) and T14(III) depend mainly on the floe aspect ratio, $d_{l v l} / \ell_{f}$, while differences in $C_{k}$ depend on the ridging intensity, $h_{k} / \ell_{k}$. As shown in Figures 9a and $9 \mathrm{~d}$, neither of these ratios is well predicted by the parameterizations of ice geometry (Equations 13 and 14), with parameterizations 
overestimating the results in both cases. For the highest values of ridging intensity $\left(h_{k} / \ell_{k} \gtrsim 5 \times 10^{-2}\right)$ predicted values fall near the one-to-one line but deviate substantially as observed values decrease (Figure 9a). As such, the overall magnitude of $C_{k}$ values is not strongly modified by the over-prediction of ridging intensity, but the decreased range of variability of modeled values is responsible for the muted seasonality of $C_{k}$ seen in the T14(III) scheme. Considering the separate roles of $h_{k}$ and $\ell_{k}$ in setting this ratio, the predictions of each individual variable have as much (or more) variability as observations (Figure 9b), but there is an apparent compensating effect between the two quantities. Predicted values of $h_{k}$ and $\ell_{k}$ vary roughly along lines of constant $h_{k} / \ell_{k}$, while observations vary primarily across lines of $h_{k} / \ell_{k}$.

The elevated levels of $C_{f}$ seen in the T14(III) test result from parameterized values of the aspect ratio, $d_{l v l} / \ell_{f}$, being much greater than observations across nearly the full range of values (Figure $9 \mathrm{~d}$, black points), with a median factor of $\sim 4$ times higher than the observed values. Differences between the observed and predicted aspect ratio are driven solely by differences in $\ell_{f}\left(d_{l v l}\right.$ is not parameterized), which is generally underestimated by Equation 14 (Figure 9c). The relationship between floe lengths and ice concentration used in Equation 14 to predict $\ell_{f}$ is an empirical result derived from a set of aerial photos of ice in the marginal ice zone in the Fram Strait (Lüpkes, Gryanik, Hartmann, \& Andreas, 2012). However, a wide variety of factors set the size and density of floes (Roach et al., 2018) and so it is unlikely that such empirical relationships would be valid in different Arctic regions and all times of year. The mismatch in the seasonality of $C_{i o}$ between observations and values predicted with the T14(III) parameterization arise mainly from this overestimate of aspect ratio. A modification to the parameters used in Equation 14 (to $\ell_{f, \min }=18.4 \mathrm{~m}, \ell_{f, \max }=1,730 \mathrm{~m}$, $b_{2}=0.9$ ) provided a much better to fit the floe length observations (Figures $9 \mathrm{c}$ and $9 \mathrm{~d}$, gray points). However, the applications of the T14(III) scheme using the modified parameters in Equation 14 still retained the seasonal mismatch in $C_{i o}$ (not shown), albeit to a lesser degree (possibly due to the very wide variability around the fitted curve in Figure 9c, noting that the comparisons in Figure 9d are plotted on logarithmic axes).

\section{Discussion}

\subsection{Comparison With Previous Drag Observations}

The range of values reported for the ice-ocean drag coefficient are consistent with previous observations. Shirasawa and Ingram (1991) and Lu et al. (2011) collated observations of the ice-ocean drag coefficient from a wide set of historical studies (publication dates from 1970 to 1997). These studies indicate a broad range of measured values with extremes from as low as $0.13 \times 10^{-3}$ (under land-fast ice in Hudson's bay; Shirasawa et al., 1989) to the highest value of $47 \times 10^{-3}$ (indirectly estimated based on fitting log-layer profiles to velocity measurements; Johannessen, 1970). The bulk of the studies summarized suggest drag coefficient values range from roughly 1 to $20 \times 10^{-3}$. More modern studies based either on direct measurements (Cole, Timmermans, et al., 2014; Cole, Toole, et al., 2017; Randelhoff et al., 2014; Shaw et al., 2008) or force-balance approaches (Dewey, 2019; Heorton, Tsamados, et al., 2019; T. W. Kim et al., 2017; Randelhoff et al., 2014) provide similar limits. This study finds drag coefficient values from 1.3 to $12.3 \times 10^{-3}$, which fall well within the conventional bounds, and the mean and median values are close to, but slightly below, the canonical drag coefficient value of $5.5 \times 10^{-3}$ (Figure 10). The overall mean value of $4.6 \times 10^{-3}$ in these observations is very similar to the average ice-ocean drag coefficient of $4.7 \times 10^{-3}$ found by Dewey (2019) for the Beaufort Sea.

Cole, Toole, et al. (2017) present detailed analysis of surface momentum flux from four ice drift stations in the Beaufort Sea, each containing a cluster of autonomous instruments. The four clusters provide measurements spanning March-December 2014, nearly a full annual cycle. Their results show weekly median ice-ocean drag coefficients ranging from $\sim 0.2$ to $10 \times 10^{-3}$, with significant spatial and temporal variability (see their Figure 12). Their measured values of $C_{i o}$ span a broader range than reported here, with minimum values an order-of-magnitude lower than ours (but similar maximum values). Nonetheless, there is good agreement with some of the qualitative behavior exhibited by the ice cluster measurements. Namely, despite strong spatial variation in the values of $C_{i o}$, all of the ice clusters showed consistent seasonal variations in ice-ocean drag, with minimum values at the time of ice minimum (August to September) and maximum values in spring (April to June). Dewey (2019) find a similar seasonal cycle based on a force-balance approach to calculate $C_{i o}$ from remote measurements in the Beaufort Sea over a 5-year period from 2011 to 2016: Basin-wide average $C_{i o}$ show minimum values from July to October of each year. These patterns are 


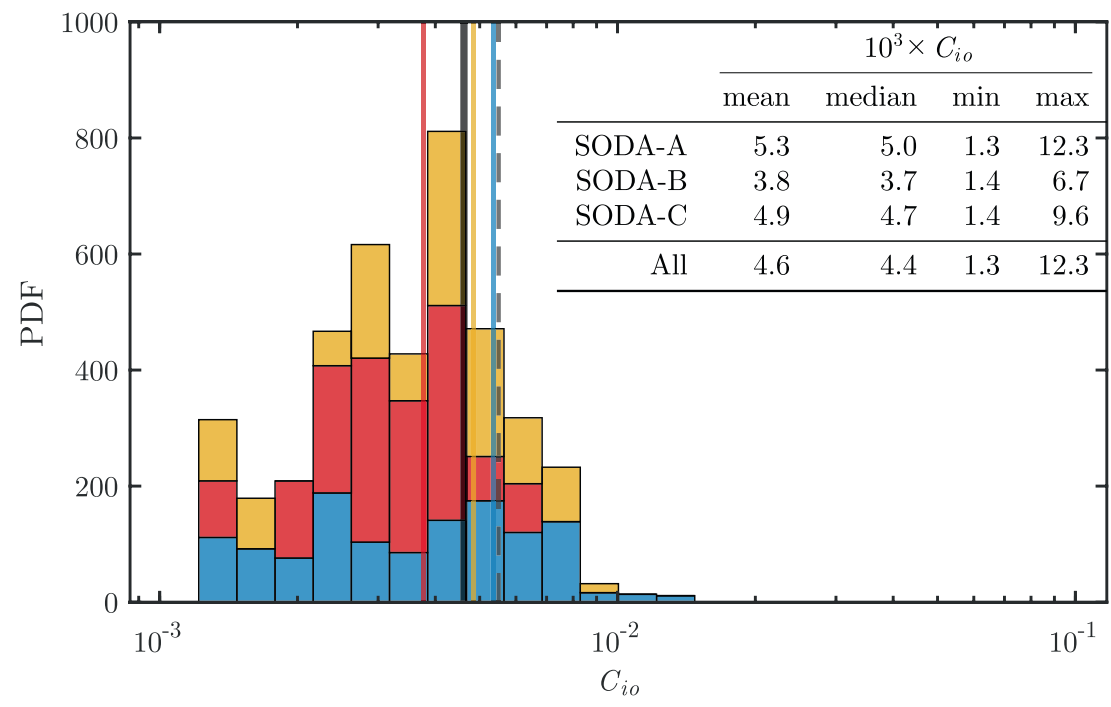

Figure 10. Stacked histograms showing the probability distribution function (PDF) of the ice-ocean drag coefficient values calculated at each of the three moorings (colored by mooring according to Figure 2a). Colored vertical lines show the annual mean value of $C_{i o}$ for each mooring, and the vertical black line shows the overall mean. The vertical gray dashed line shows the value of $C_{i o}=5.5 \times 10^{-3}$ for comparison.

in agreement with our observations which show minimum ice-ocean drag coefficient values in fall (Figure 6). In contrast, pan-Arctic averages of $C_{i o}$ from models incorporating a variable drag coefficient scheme (Section 2.1) show the opposite behavior (Martin, Tsamados, et al., 2016; Tsamados et al., 2014). In those models, the maximum value of $C_{i o}$ occurs during the summer/fall season, driven by form drag on floe edges (Equation 9a). As described above (Section 4.3), seasonality in modeled values of $C_{i 0}$ may be a result of over predicted values of the floe aspect ratio, $d_{l v l} / \ell_{f}$.

With a few exceptions, direct observational estimates of the ice-ocean drag coefficient are made using point measurements of turbulent fluxes. In comparison to the force-balance approach used here, $C_{i o}$ values derived from point measurements require far fewer assumptions about the ice dynamics (e.g., they are valid whether or not the ice is in free drift). However, these measurements are also inherently local and as such it is not clear how they scale to application across entire ice floes. For logistical reasons, measurements are typically made away from ice keels, so reported values of $C_{i o}$ may under-represent floe- or regional-average values (McPhee, 2012). Randelhoff et al. (2014) provide a direct comparison between a force-balance approach to calculate ice-ocean drag (the procedure used here) and in situ measurements of turbulent fluxes. Their results showed that the force-balance approach produced ice-ocean stress estimates that were, on average, 3 times larger than direct measurements. They attribute the mismatch to unmeasured sources of drag (e.g., due to internal wave radiation; McPhee \& Kantha, 1989), but it may also be due to horizontally varying and thus non-local turbulence. Similarly, application of the force-balance approach to the ice cluster data from Cole, Toole, et al. (2017) shows higher values of $C_{i o}$ and decreased temporal variability compared to local measurements (Heorton, Tsamados, et al., 2019). While this may explain why the values of $C_{i o}$ observed here have a much higher minimum value than those by Cole, Toole, et al. (2017), more work is needed to understand the inherent differences between direct point measurements and force-balance measurements of ice-ocean drag.

In comparing values of $C_{i o}$ between different studies, it is important to consider the choice of reference depth used, which will impact the drag coefficient through depth variations of $\boldsymbol{u}_{0}$. For example, repeating our analysis with a shallower reference depth of $z_{\text {ref }}=6 \mathrm{~m}$ yields slightly higher values of $C_{i o}$, with an overall average of $5.2 \times 10^{-3}$ (compared to $4.6 \times 10^{-3}$ for $z_{\text {ref }}=10 \mathrm{~m}$ ). Typically, values of $C_{i o}$ are reported corresponding to either fixed reference depths near the ice bottom, thus in or near the logarithmic boundary layer, or they are reported using the underlying geostrophic current, $\boldsymbol{u}_{g}$, as a reference velocity (Table 1 in Lu et al. [2011], lists reference depths used for a number of studies). Within the log-layer, $\boldsymbol{u}_{o} \propto \boldsymbol{u}_{*}$, so the application of the quadratic drag law is appropriate. However, beyond the logarithmic layer, the rela- 
tionship between stress and velocity in the ice-ocean boundary layer is not expected to be quadratic (e.g., McPhee, 2008, and references therein). If $\boldsymbol{u}_{g}$ is used as a reference velocity, drag may be better described by Rossby Similarity Theory (Blackadar \& Tennekes, 1968; McPhee, 2008), which accounts for the existence of an outer Ekman-like layer matched to an inner logarithmic layer (as has been observed in the ice-ocean boundary layer, e.g., Hunkins, 1966; McPhee, 1979). In this more general case, McPhee (1979, and others) find reasonable empirical agreement from an alternative power law form: $\left|\tau_{i o}\right| \propto\left|\boldsymbol{u}_{i}-\boldsymbol{u}_{g}\right|^{n}$ where $n<2$ (e.g., Cole, Toole, et al., 2017, find values of $n$ ranging from 0.51 to 1.76). The use of a fixed reference depth of $z_{\text {ref }}=10 \mathrm{~m}$ in the present study likely extends beyond the surface log-layer so the quadratic drag law is not strictly applicable. Nonetheless, tested parameterizations that assume a law-of-the-wall velocity profile (T14(I), T14(II)) produce reasonable results (Figures 6 and 7). Furthermore, the relationship between stress and relative velocity seems to be well described by the quadratic drag law (Figure 3). This suggests a "fuzzy" transition between the inner logarithmic boundary layer and the outer Ekman-like layer such that the lawof-the-wall still provides a useful approximation for determining $C_{i o}$. Likely, the use of a smaller reference depth that is closer to the base of the logarithmic boundary layer may increase the accuracy of the quadratic drag assumption (e.g., Park \& Stewart, 2016, suggest a hybrid Rossby Similarity Theory using the quadratic drag law to model the inner boundary layer coupled to classic Ekman-layer dynamics for the outer layer).

\subsection{Recommendations for Model Development}

This study identifies some possible directions that future modeling work could focus on. The parameterizations here can be described as having two parts: one part that models the underlying physics (Equation 9; tested by schemes L11, T14(I), T14(II)), and a second part for the geometry (Equations 13 and 14; tested by scheme T14(III)). There are some opportunities for improvements in both of these parts; however, based on the results in Section 4.3, it is apparent that there is a more urgent need to improve descriptions of the sea ice geometry.

Translating bulk sea ice model outputs to the detailed geometry needed to apply Equations 9a-9c appears to be a particular challenge. Both the ridging intensity, $h_{k} / \ell_{k}$, and the floe aspect ratio, $d_{l v l} / \ell_{f}$, are overpredicted by the parameterization schemes from Section 2.2 (see Figure 9). Some efforts are being made to directly model different aspects of the sea ice geometry (e.g., floe sizes, Roach et al., 2018; or keel statistics, Roberts et al., 2019), thus alleviating the need for geometry parameterizations. However, until such modeling schemes are widely implemented, there will be some value in improvements to existing geometry parameterizations.

The keel depth and spacing predicted from model outputs by Tsamados et al. (2014) (Equations 13a and 13b) are based on geometric arguments that are informed by measurements of sea ice sails and keels. In formulating those equations, the authors assume a uniform field of equally sized, shaped, and spaced non-overlapping ridges in each grid cell box. However, past measurements have shown that keel depth, width, and spacing are better described by statistical distributions (e.g., Davis \& Wadhams, 1995; Hibler et al., 1972; Martin, 2007; Timco \& Burden, 1997; Wadhams \& Davy, 1986). Some improvement in the parameterizations could likely be made simply by considering the shape of these distributions. For example, using an exponential distribution to describe relative keel depths (per Wadhams \& Davy, 1986), the total ice volume associated with keels will differ from that calculated with a uniform distribution by a factor of 2 when both distributions have the same mean keel depth. Figure $9 \mathrm{~b}$ suggests that some of these geometry variables may be jointly distributed.

The mismatch between modeled and observed seasonal variations in ice-ocean drag coefficients is largely due to discrepancies in modeled floe lengths, $\ell_{f}$ (Section 4.3). The floe length parameterization (Equation 14) is an empirical result relating $\ell_{f}$ to $A$ from aerial photographs of ice in the Fram Strait in the 1990s (Lüpkes, Gryanik, Hartmann, \& Andreas, 2012). While Lüpkes, Gryanik, Hartmann, and Andreas (2012) developed this relationship for the marginal ice zone, its implementation by Tsamados et al. (2014) does not distinguish between marginal and pack ice (though the authors acknowledge a possible breakdown in winter conditions). Additionally, Lüpkes, Gryanik, Hartmann, and Andreas (2012) highlight that variability in the relationship between $\ell_{f}$ and $A$ points to other variable dependencies on floe sizes. Employed here, we are able to adjust the input parameters to Equation 14 to provide a better fit to the observed floe lengths (Figure 9c), suggesting that some general variability in the behavior of floe length may be modeled by some 
form of that equation. However, there remains a significant amount of scatter in the observations (over orders of magnitude), and so even with the adjusted parameters the model floe edge drag is still too high during the ice melt season. Future development of empirically derived floe length parameterizations should, at minimum, include observations from across different Arctic regions based on modern ice conditions. Moreover, deterministic models for the evolution of floe size distributions (Horvat \& Tziperman, 2017; Roach et al., 2018) highlight which other variables could be included in empirical fits. For example, rather than casting $\ell_{f}$ as a function just of $A$, it may be more appropriate to develop two-parameter empirical fits that also include the ice thickness. Further multi-parameter fits might consider the inclusion of wind speed, $\left|\boldsymbol{u}_{a}\right|$ (which would have impacts both on sea ice welding and breakup by driving ice motion, and on surface wave conditions which can lead to fracture), and sea surface temperature (which is important for lateral growth and melt).

While better geometry schemes should be a focus, the improvement of the T14(II) scheme over the L11 and T14(I) schemes also show that minor modifications to the physics part of the parameterization scheme have the potential to increase the predictive skill. There are a number of changes between the schemes (see Table 2), however, most of the improvement is made by simply choosing more appropriate values of the coefficients of resistance $c_{f}$ and $c_{k}$. While those are chosen here with a slightly ad hoc fitting method (using multiple linear regression; see Section 3.4), determining appropriate ranges for these values is a subject of ongoing research (e.g., Zu et al., 2020). In addition to these coefficients, the parameterization schemes tested include a number of other constants whose values are not fully constrained that could be used to tune the modeled drag coefficients: $c_{s}, s_{l}, z_{0 w}, z_{0 i}, m_{w}$.

Detailed optimization accounting for all free parameters or more rigorously fitting the values of $c_{f}$ and $c_{k}$ is deliberately not performed here. This choice is primarily driven by the fact that the tests here do not account for all of the physical processes that modify the ice-ocean drag coefficient. In particular, the parameterization schemes only model the neutral drag coefficient and do not account for variations due to buoyancy (which should be included as a correction term; e.g., Lüpkes \& Gryanik, 2015), whereas the observed values of $C_{i o}$ reflect the total drag, including non-neutral effects. Similarly, shallow surface stratification may act to partly decouple the sea ice motion from subsurface velocity measurements, especially during the melt season. Additionally, drag due to internal wave radiation is thought to be important in some oceanographic conditions (McPhee \& Kantha, 1989; Pite et al., 1995) but is not included. Finally, the forms of the functions $P_{0}$ (Equation 11) and $c_{s}$ (Equation 12) are based on an assumed velocity profile that may not be suitable through the full reference depth; the logarithmic boundary layer at the ice-ocean interface is thought to be only 2 m thick (e.g., Cole, Toole, et al., 2017; McPhee, 2002; Randelhoff et al., 2014; Shaw et al., 2008), which is much shallower than the 10-m reference depth used. The generally close match between parameterized values of $C_{i o}$ (with T14(II)) and those determined through the force balance suggest that these effects may be small, but a thorough optimization of free parameters should be performed that considers these effects.

In addition to improvements in existing parameterizations, there has been some interest in simplified parameterization schemes for drag coefficients based solely on ice concentration (which have been applied for atmospheric drag; e.g., Andreas, Horst, et al., 2010a; Andreas, Persson, et al., 2010b; Lüpkes, Gryanik, Rösel, et al., 2013). While there is some value in such an approach, we recommend caution in the development of such schemes for the ice-ocean drag coefficient. The atmospheric drag schemes such as those by Andreas, Horst, et al. (2010a) focus on the effects of floe edges, and thus might work well when the sea ice concentration dominates form drag but less well when drag is dominated by ice ridges. Because of the different scales of both the boundary layer and the ridges at the ice-ocean boundary compared to the atmosphere-ice boundary, the influence of keels on ice-ocean drag may be much more important than the influence of ice sails on atmospheric drag. Thus, approaches for simplified modeling employed in atmospheric literature may not be appropriate to adopt for ice-ocean drag. The differing timescales for ridge intensity growth (relatively slow; Figure 5e) compared to ice concentration growth (relatively rapid; Figures 2d and 2e), along with the strong control of ridging intensity on the total ice-ocean drag (Section 4.3) means that concentration-based schemes are unlikely to be capable of representing ice-ocean drag. From the results of this study, we speculate that a simplified ice-ocean drag parameterization might be better described with a two-parameter scheme that includes both ice concentration and ice thickness. 


\subsection{Implications for Momentum Transfer Into the Ocean}

We have focused on the efficiency of momentum transfer between the sea ice and the upper ocean; however, these questions exist in a broader context of the impact of sea ice on mediating total momentum flux between the ocean and the atmosphere. Conventional wisdom has been that sea ice damps atmosphere-ocean momentum flux (Plueddemann et al., 1998; Rainville \& Woodgate, 2009), and so an increase in open water will lead to an increase in momentum flux into the ocean (Rainville et al., 2011). However, other recent studies have suggested a more complex view (Dosser \& Rainville, 2016; Martin, Steele, \& Zhang, 2014; Martin, Tsamados, et al., 2016). Martin, Steele, and Zhang (2014) and Martin, Tsamados, et al. (2016) show that sea ice can either enhance or diminish momentum flux into the ocean depending on the interplay between internal ice stress and wind stress (which is amplified over the sea ice; e.g., Guest et al., 1995, and many others). A detailed accounting of the upper ocean response to the combined sea ice and atmospheric forcing is outside the scope of the current study; here we consider the potential for amplification or damping of momentum flux into the ocean by sea ice.

The equivalent drag coefficient, $C_{\text {equiv }}$ (Equation 7) provides a measure of the total momentum transfer efficiency between the atmosphere and the ocean as it is mediated by sea ice. To provide additional context for the observations, consider two limits for the value of $C_{\text {equiv }}$ : (1) a "free-drift limit", where $\boldsymbol{F}_{a}=\boldsymbol{F}_{i}=0$ in Equation 6, so $\tau_{\text {ocn }}=\tau_{a t m}$; (2) the atmosphere-ice stress, $\tau_{a i}$, is balanced by internal ice stress, $\nabla \cdot \sigma$, and $\boldsymbol{F}_{a}$ is negligible, so $\tau_{i o}=0$. Then for each case the equivalent drag coefficient is given by:

$$
\begin{array}{ll}
\text { case 1: } & C_{\text {equiv }}=A C_{a i}+(1-A) C_{a o}, \\
\text { case 2: } & C_{\text {equiv }}=(1-A) C_{a o} .
\end{array}
$$

Taking $C_{a o}$ as constant (an appropriate approximation for typical wind speeds), the two cases above provide formula for $C_{\text {equiv }}$ that are functions solely of ice concentration (noting application of an ice-concentration based parameterization scheme for $C_{a i}$ ). While these two cases are referred to as limits, they are not strict limits as both the role of acceleration terms $\left(\boldsymbol{F}_{a}\right)$ and the vector addition of terms in Equation 6 can either increase or decrease $C_{\text {equiv }}$ beyond these bounds.

Values of $C_{\text {equiv }}$ span a wide range, and the variability of observed values increases with increasing sea ice concentration (Figure 11). This increase in variability of $C_{\text {equiv }}$ with $A$ reflects the divergence of the two limits of $C_{\text {equiv }}$ introduced above, which both approach $C_{a o}$ as $A \rightarrow 0$ but either increase (Equation 15a) or decrease (Equation 15b) as $A$ increases. Results also show a separation of $C_{\text {equiv }}$ based on the wind factor $\left(\left|\boldsymbol{u}_{i}\right| /\left|\boldsymbol{u}_{a}\right|\right)$. Points with a wind factor $\geq 2 \%$ (defined as being in free drift) generally fall near the upper "freedrift limit" (as expected). This limit shows that in the absence of acceleration terms $\left(\boldsymbol{F}_{a}\right)$, ice in free drift will amplify the efficiency of stress transfer compared to open water; however, as $\boldsymbol{F}_{a}$ also includes the Coriolis acceleration, $\boldsymbol{F}_{a}$ is non-zero even at steady-state, introducing some additional variability. Points with wind factor below $2 \%$ cover a more broad range of values, but for low values (wind factor $\leq 1 \%$ ), $C_{\text {equiv }}$ are generally bounded by Equation 15b. This shows that, as expected, the ice interaction force $\boldsymbol{F}_{i}$ causes a reduction in momentum transfer relative to open-water conditions. Whether the net effect of the ice is to amplify or damp momentum transfer ultimately depends on the strength of this force.

Annual median values of $C_{\text {equiv }}$ were similar for each of the three mooring locations with a slight northsouth trend: $1.69,1.44,1.34 \times 10^{-3}$ for SODA-A, -B, and -C, respectively. This similarity reflects that increased open-water areas (which have a lower efficiency of momentum transfer) at the southern moorings may partly offset expected increases in winter $C_{\text {equiv }}$ due to free-drift conditions. However, because wind forcing also has strong seasonal variations with a winter maximum (e.g., Dosser \& Rainville, 2016), longterm trends in the total momentum flux into the ocean $\left(\tau_{\text {ocn }}\right)$ will depend both on a balance of increasing open-water conditions and changing internal stress conditions in the winter.

Based on the $2 \%$-rule, the wind factor $\left(\left|\boldsymbol{u}_{i}\right| /\left|\boldsymbol{u}_{a}\right|\right)$ provides a first-order estimate of the extent of free drift conditions at each mooring. While only a rule-of-thumb, measured values of the wind factor showed asymptotic behavior supporting use of this rule: as the wind speed increased (i.e., as $\tau_{a i}$ becomes a dominant term in the force balance), wind factor values converged around $2 \%$; bin-average values of the wind fac- 


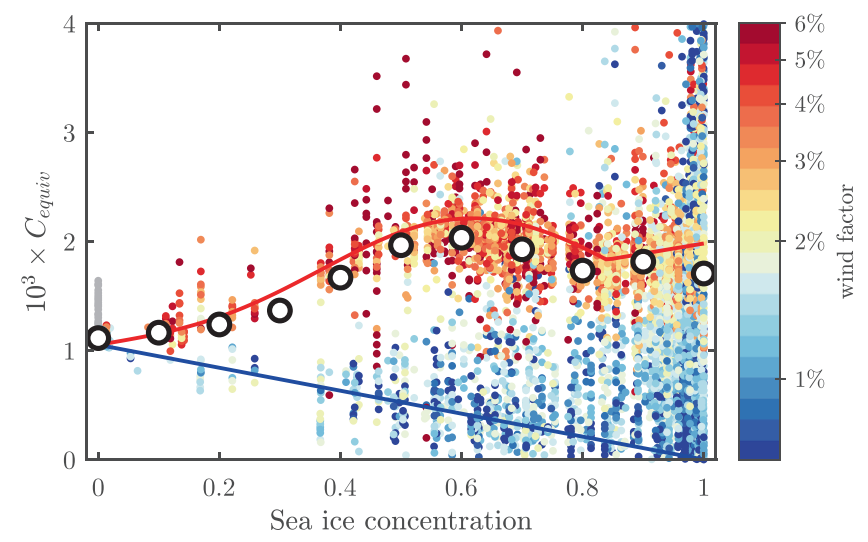

Figure 11. Equivalent drag coefficient $C_{\text {equiv }}$ (Equation 7) as a function of sea ice concentration (from ERA5). Points shows all hourly values from all moorings, colored by wind factor (log-scale; gray points had no measurable $\boldsymbol{u}_{i}$ ), while black circles show bin-median values by sea ice concentration. The red and blue lines shows the limit cases discussed in the text: red is Equation 15a; blue is Equation 15b. tor stay approximately near $2 \%$ across a wide range of wind speeds (Figure 12a). There was also a relationship between wind factor and sea ice concentration: For concentrations below $\sim 80 \%-85 \%$, the wind factor was elevated and generally $>2 \%$ (Figure $12 \mathrm{~b}$ ). This suggests that an $80 \%-85 \%$ ice-concentration-based limit for defining free drift is an approximation of the $2 \%$-rule, but it may be the case that free drift conditions also occur intermittently for higher ice concentrations (e.g., on short timescales, atmospheric stress may be balanced primarily by only one of either the ice-ocean or ice-ice stresses, as in Steele, Zhang, et al. [1997]). The prevalence of wind factor values greater than $2 \%$ have a north-south trend, with roughly $66 \%$ of measurements designated as being free drift at SODA-A, 54\% at SODA-B, and 37\% at SODA-C. Dosser and Rainville (2016) previously showed that the wind factor is a useful indicator for atmosphere-ice-ocean momentum transfer. If the differences between SODA-A and SODA-C are indicative of future trends of sea ice (in which more and more of the Arctic is similar to SODA-A) then this suggests the potential for increasing amplification of stress transfer from the atmosphere to the ocean in the Beaufort Sea during winter.

Martin, Steele, and Zhang (2014) and Martin, Tsamados, et al. (2016) suggests that interplay between wind stress enhancement over sea ice and internal ice stresses (i.e., the relative sizes of $\boldsymbol{\tau}_{a t m}$ and $\boldsymbol{F}_{i}$ in Equation 6) lead to a local maximum in the normalized $\tau_{\text {ocn }}$ at some optimal sea ice concentration (their results suggest $\sim 80 \%-90 \%$ ). We see similar evidence for an optimal sea ice concentration in $C_{\text {equiv }}$; binned-median values of $C_{\text {equiv }}$ have a peak near $60 \%$ ice concentration (Figure 11). However, our observations show that binned-median $C_{\text {equiv }}$ roughly follow the free-drift limit (Case 1), and there is not an appreciable decrease below that limit in median $C_{\text {equiv }}$ at $100 \%$ ice concentration (which is in contrast to the pan-Arctic average results presented by Martin, Steele, and Zhang [2014]). This suggests that the optimal ice concentration for momentum transfer seen in our results is driven by the maximum of Equation 15a, and is minimally affected the ice interaction force $\left(\boldsymbol{F}_{i}\right)$. As such, results for optimal ice concentration will be highly sensitive to the parameterization of $C_{i a}$. Furthermore, these results indicate that, on average, at all three moorings the presences of sea ice cause an amplification of stress transfer compared to open-water conditions for a given wind speed. This is consistent with Martin, Tsamados, et al. (2016), who found that sea ice in the Beaufort Sea causes a mean amplification of stress into the ocean for all seasons regardless of whether a constant or variable ice-ocean drag coefficient was used in the model (see their Figure 12).

\section{Conclusions}

Using a force-balance approach to estimate the ice ocean drag coefficient, $C_{i o}$, the annual cycle of the efficiency of ice-ocean momentum transfer is inferred from mooring observations. These estimates compare favorably with drag coefficients using parameterization schemes, based on measured statistics of ice geometry, as well as with previous observations of ice-ocean drag. We summarize the main contributions of the study as follows:

1. The ice ocean drag coefficient, $C_{i o}$, varied seasonally. Variations were more pronounced for the moorings in the seasonal ice zone compared to the mooring that was ice-covered through the full year (Figure 6), suggesting that the enhanced seasonality of the Arctic ice pack is directly influencing seasonality in $C_{i o}$. This manifested as a decrease in $C_{i o}$ in the summer and fall, driven by changes in intensity of ridged ice (Figure 8). Wintertime mean values of $C_{i o}$ were similar to, or higher than, the canonical value of $5.5 \times 10^{-3}$ (up to a maximum of $12.3 \times 10^{-3}$ ), but summer and fall values at SODA-A and -B (which may be more representative of future conditions) were as low as $\sim 1.3 \times 10^{-3}$ (Figure 10). The observed seasonality agrees with previous observational studies in the Western Arctic (Cole, Toole, et al., 2017; Dewey, 2019), but contrast with pan-Arctic model results (Martin, Tsamados, et al., 2016; Tsamados et al., 2014) 

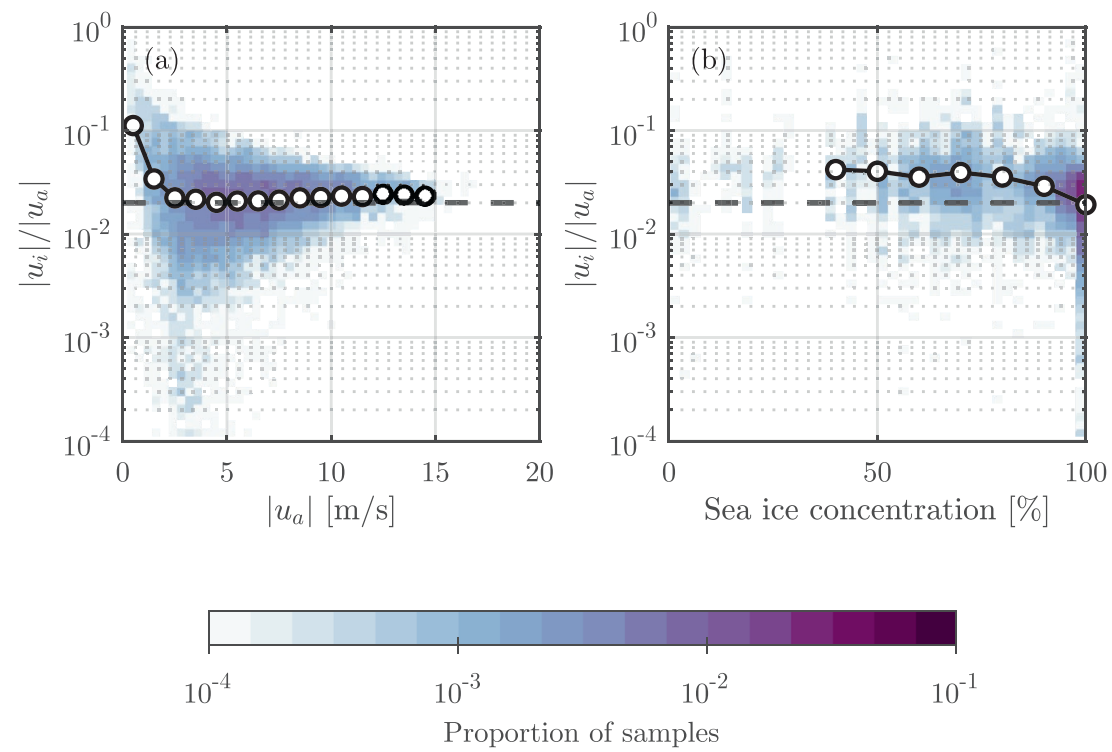

Figure 12. Wind factor $\left(\left|\boldsymbol{u}_{i}\right| /\left|\boldsymbol{u}_{a}\right|\right)$ as a function of (a) wind speed, and (b) sea ice concentration (from ERA5). In both panels, shading shows a 2-dimensional histogram of the proportion of total samples (on a log-scale), while black lines with circles show the values of wind factor bin-averaged by (a) wind speed, and (b) sea ice concentration. Binaverages in (b) were only produced for sea ice concentration $\geq 40 \%$ due to data scarcity for lower ice concentrations. The horizontal dashed black line in both panels corresponds to a wind factor of $2 \%$.

2. Geometry-based drag parameterizations reproduce many of the spatial and temporal variations of iceocean drag, provided that the ice geometry is known (Figures 6 and 7). Slight modifications to the existing parameterization schemes produces the most favorable results (T14(II); Figure 7c), but a full optimization of all free parameters has yet to be performed (and should account for non-neutral conditions and differences in boundary layer structure). Parameterization of the ice geometry (T14(III)) appears more challenging (Figure 7d), particularly predicting the correct floe sizes (impacting the total floe edge drag, Figures 8g-8i). The mismatch in seasonality of ice-ocean drag between observations (Cole, Toole, et al., 2017; Dewey, 2019, and the present study) and models (Martin, Tsamados, et al., 2016; Tsamados et al., 2014) is likely a direct result of the difficulties in predicting floe aspect ratios using bulk parameters. Despite these challenges, the scheme that included ice geometry parameterization (T14(III)) still provided reasonably predictions of the ice-ocean drag prior to ice breakup in the spring/summer (Figure 6, red lines)

3. In the seasonal ice zone, ridging intensity grows relatively slowly compared to the growth of ice concentration (compare Figures $2 \mathrm{~d}$ and $2 \mathrm{e}$ with Figure $5 \mathrm{f}$ ). As a result, approaches for simplified ice concentration-based parameterization schemes that have been successful for calculating atmospheric drag (e.g., Andreas, Horst, et al., 2010a; Andreas, Persson, et al., 2010b) may not be the correct approach for drag at the ice-ocean interface. It is unlikely that schemes based solely on ice concentration will be able to adequately capture variations in ice-ocean drag during the ice growth season

4. The presence of sea ice causes a net amplification of the efficiency of stress input to the ocean compared to open water (Section 5.3) which we attribute to the prevalence of free drift conditions (including intermittently during full ice cover). Our measurements support the notion of an "optimal ice concentration" for momentum transfer (Martin, Steele, \& Zhang, 2014; Martin, Tsamados, et al., 2016), but suggest the value of the optimal concentration has high sensitivity to the parameterization of the atmosphere-ice drag coefficient, $C_{a i}$ (Figure 11). A comparison between moorings indicates that free drift conditions are more common to the south, and thus may become more common throughout the Beaufort Sea in the future, with a net trend of amplified coupling between the atmosphere and the ocean

The capability of models to represent the coupled atmosphere-ice-ocean system continues to evolve. Despite mismatches in predictions of ice geometry statistics which are used as inputs, the general success of the parameterization schemes described here gives greater confidence in our ability to use modeled results 
to learn about the "new Arctic", provided that methods can be developed to account for those mismatches. New sea-ice modeling schemes may be able to directly represent floe size distributions (Roach et al., 2018) or keel statistics (Roberts et al., 2019), reducing the need to redefine parameterizations of sea ice geometry. As model parameterizations of ice-ocean drag evolve, it will become important for users who apply those schemes to choose a framework that matches the model application, including an appropriate choice of reference depth, $z_{\text {ref }}$ For example, for an upper-ocean mixing study that uses $\tau_{i o}$ as a surface boundary condition it may be most appropriate to use a value of $C_{i 0}$ consistent with drag at the base of the surface log-layer, or to choose $z_{\text {ref }}$ in Equation 9 corresponding to the shallowest resolved ocean model level. Drag in a large-scale ice drift model driven by geostrophic ocean currents may be better described by Rossby Similarity Theory (Blackadar \& Tennekes, 1968; McPhee, 2008) than by a quadratic drag law; though linking the "effective" roughness length used in that theory to statistics of large scale geometric features remains an open problem. Finally, differences between drag values measured at the different mooring sites indicates that variations in ice morphology may lead to large-scale spatial gradients in the ice-ocean drag, and consequently the surface momentum flux into the ocean, which may have important consequences for studies of large-scale Beaufort Sea circulation (e.g., gyre equilibrium and freshwater storage; Armitage, Manucharyan, et al., 2020; Meneghello et al., 2018; Timmermans et al., 2018).

\section{Data Availability Statement}

Data files containing the timeseries of the measurements and results described in this study, including sea ice momentum terms, sea ice geometry, and ice-ocean drag coefficients, are available at http://hdl.handle. net/1773/46919. More information about the project can be found at www.apl.washington.edu/soda.

\section{Acknowledgments}

This work was supported by the Office of Naval Research as part of the Stratified Ocean Dynamics of the Arctic (SODA) research project. Funding was through grant numbers N00014-16-12349, N00014-14-1-2377, N00014-181-2687, and N00014-16-1-2381. The authors thank Captain Greg Tlapa and Captain MaryEllen Durley, along with the rest of the command team and crew of USCGC Healy for operational support in 2018 and 2019. This work has benefited from ideas and feedback from members of the SODA project team and by two anonymous reviewers. The authors would also like to thank Sarah Dewey for helpful views and conversations.

\section{References}

Andreas, E. L. (2011). A relationship between the aerodynamic and physical roughness of winter sea ice. Quarterly Journal of the Royal Meteorological Society, 137(659), 1581-1588. https://doi.org/10.1002/qj.842

Andreas, E. L., Horst, T. W., Grachev, A. A., Persson, P. O. G., Fairall, C. W., Guest, P. S., \& Jordan, R. E. (2010a). Parametrizing turbulent exchange over summer sea ice and the marginal ice zone. Quarterly Journal of the Royal Meteorological Society, 136(649), 927-943. https://doi.org/10.1002/qj.618

Andreas, E. L., Persson, P. O. G., Grachev, A. A., Jordan, R. E., Horst, T. W., Guest, P. S., \& Fairall, C. W. (2010b). Parameterizing turbulent exchange over sea ice in winter. Journal of Hydrometeorology, 11(1), 87-104. https://doi.org/10.1175/2009JHM1102.1

Armitage, T. W. K., Bacon, S., Ridout, A. L., Petty, A. A., Wolbach, S., \& Tsamados, M. (2017). Arctic Ocean surface geostrophic circulation 2003-2014. The Cryosphere, 11(4), 1767-1780. https://doi.org/10.5194/tc-11-1767-2017

Armitage, T. W. K., Manucharyan, G. E., Petty, A. A., Kwok, R., \& Thompson, A. F. (2020). Enhanced eddy activity in the Beaufort Gyre in response to sea ice loss. Nature Communications, 11(1), 761. https://doi.org/10.1038/s41467-020-14449-z

Arya, S. P. S. (1975). A drag partition theory for determining the large-scale roughness parameter and wind stress on the Arctic pack ice. Journal of Geophysical Research, 80(24), 3447-3454. https://doi.org/10.1029/JC080i024p03447

Belliveau, D. J., Bugden, G. L., \& Melrose, S. G. K. (1989). Measurement of sea ice motion using bottom mounted Acoustic Doppler Current Profilers. Sea Technology, 30.

Bendat, J. S., \& Piersol, A. G. (1971). Random data: Analysis and measurement procedures. Wiley-Interscience.

Blackadar, A. K., \& Tennekes, H. (1968). Asymptotic Similarity in Neutral Barotropic Planetary Boundary Layers. Journal of the Atmospheric Sciences, 25(6), 1015-1020. https://doi.org/10.1175/1520-0469(1968)025〈1015:ASINBP〉2.0.CO;2

Brenner, S., Rainville, L., Thomson, J., \& Lee, C. (2020). The evolution of a shallow front in the Arctic marginal ice zone. Elementa: Science of the Anthropocene, 8(17), 17. https://doi.org/10.1525/elementa.413

Castellani, G., Gerdes, R., Losch, M., \& Lüpkes, C. (2015). Impact of sea-ice bottom topography on the Ekman Pumping. In G. Lohmann, H. Meggers, V. Unnithan, D. Wolf-Gladrow, J. Notholt, A. Bracher (Eds.), Towards an interdisciplinary approach in Earth system science: Advances of a Helmholtz Graduate Research School (pp. 139-148). Springer International Publishing. https://doi. org/10.1007/978-3-319-13865-7_16

Castellani, G., Lüpkes, C., Hendricks, S., \& Gerdes, R. (2014). Variability of Arctic sea-ice topography and its impact on the atmospheric surface drag. Journal of Geophysical Research: Oceans, 119(10), 6743-6762. https://doi.org/10.1002/2013JC009712

Castellani, G., Losch, M., Ungermann, M., \& Gerdes, R. (2018). Sea-ice drag as a function of deformation and ice cover: Effects on simulated sea ice and ocean circulation in the Arctic. Ocean Modelling, 128, 48-66. https://doi.org/10.1016/j.ocemod.2018.06.002

Cole, S. T., Timmermans, M.-L., Toole, J. M., Krishfield, R. A., \& Thwaites, F. T. (2014). Ekman veering, internal waves, and turbulence observed under Arctic sea ice. Journal of Physical Oceanography, 44(5), 1306-1328. https://doi.org/10.1175/JPO-D-12-0191.1

Cole, S. T., Toole, J. M., Lele, R., Timmermans, M.-L., Gallaher, S. G., Stanton, T. P., et al. (2017). Ice and ocean velocity in the Arctic marginal ice zone: Ice roughness and momentum transfer. Elementa: Science of the Anthropocene, 5, 55. https://doi.org/10.1525/elementa.241

Connolley, W. M., Gregory, J. M., Hunke, E. C., \& Mclaren, A. J. (2004). On the consistent scaling of terms in the sea-ice dynamics equation. Journal of Physical Oceanography, 34(7), 1776-1780. https://doi.org/10.1175/1520-0485(2004)034<1776:otcsot>2.0.co;2

Davis, N. R., \& Wadhams, P. (1995). A statistical analysis of Arctic pressure ridge morphology. Journal of Geophysical Research, 100(C6), 10915. https://doi.org/10.1029/95JC00007

Dewey, S. (2019). Evolving ice-ocean dynamics of the western Arctic (Unpublished doctoral dissertation). University of Washington. 
Dewey, S., Morison, J., Kwok, R., Dickinson, S., Morison, D., \& Andersen, R. (2018). Arctic ice-ocean coupling and gyre equilibration observed with remote sensing. Geophysical Research Letters, 45(3), 1499-1508. https://doi.org/10.1002/2017GL076229

Dosser, H. V., \& Rainville, L. (2016). Dynamics of the changing near-inertial internal wave field in the Arctic Ocean. Journal of Physical Oceanography, 46(2), 395-415. https://doi.org/10.1175/JPO-D-15-0056.1

ECMWF (2019). Part IV: Physical processes. In IFS Documentation CY46R1. ECMWF.

Leppäranta, M. (Ed.). (2011). The drift of sea ice. Springer. https://doi.org/10.1007/978-3-642-04683-4_2

Elvidge, A. D., Renfrew, I. A., Weiss, A. I., Brooks, I. M., Lachlan-Cope, T. A., \& King, J. C. (2016). Observations of surface momentum exchange over the marginal ice zone and recommendations for its parametrisation. Atmospheric Chemistry and Physics, 16(3), $1545-1563$. https://doi.org/10.5194/acp-16-1545-2016

Garbrecht, T., Lüpkes, C., Hartmann, J., \& Wolff, M. (2002). Atmospheric drag coefficients over sea ice-validation of a parameterisation concept. Tellus A: Dynamic Meteorology and Oceanography, 54(2), 205-219. https://doi.org/10.3402/tellusa.v54i2.12129

Gelaro, R., McCarty, W., Suárez, M. J., Todling, R., Molod, A., Takacs, L., et al. (2017). The modern-era retrospective analysis for research and applications, version 2 (MERRA-2). Journal of Climate, 30(14), 5419-5454. https://doi.org/10.1175/JCLI-D-16-0758.1

Graham, R. M., Cohen, L., Ritzhaupt, N., Segger, B., Graversen, R. G., Rinke, A., et al. (2019). Evaluation of six atmospheric reanalyses over Arctic sea ice from winter to early summer. Journal of Climate, 32(14), 4121-4143. https://doi.org/10.1175/JCLI-D-18-0643.1

Guest, P. S., \& Davidson, K. L. (1987). The effect of observed ice conditions on the drag coefficient in the summer East Greenland Sea marginal ice zone. Journal of Geophysical Research, 92(C7), 6943. https://doi.org/10.1029/JC092iC07p06943

Guest, P. S., Glendening, J. W., \& Davidson, K. L. (1995). An observational and numerical study of wind stress variations within marginal ice zones. Journal of Geophysical Research, 100(C6), 10887. https://doi.org/10.1029/94JC03391

Harding, S., Kilcher, L., \& Thomson, J. (2017). Turbulence measurements from compliant moorings. Part I: Motion characterization. Journal of Atmospheric and Oceanic Technology, 34(6), 1235-1247. https://doi.org/10.1175/JTECH-D-16-0189.1

Heorton, H. D. B. S., Feltham, D. L., \& Hunt, J. C. R. (2014). The response of the sea ice edge to atmospheric and oceanic jet formation. Journal of Physical Oceanography, 44(9), 2292-2316. https://doi.org/10.1175/JPO-D-13-0184.1

Heorton, H. D. B. S., Tsamados, M., Cole, S. T., Ferreira, A. M. G., Berbellini, A., Fox, M., \& Armitage, T. W. K. (2019). Retrieving sea ice drag coefficients and turning angles from in situ and satellite observations using an inverse modeling framework. Journal of Geophysical Research: Oceans, 124(8), 6388-6413. https://doi.org/10.1029/2018JC014881

Hersbach, H., Bell, B., Berrisford, P., Hirahara, S., Horányi, A., Muñoz-Sabater, J., et al. (2020). The ERA5 global reanalysis. Quarterly Journal of the Royal Meteorological Society, 146(730), 1999-2049. https://doi.org/10.1002/qj.3803

Hibler, W. D., Weeks, W. F., \& Mock, S. J. (1972). Statistical aspects of sea-ice ridge distributions. Journal of Geophysical Research, 77(30), 5954-5970. https://doi.org/10.1029/JC077i030p05954

Holton, J. R. (2004). An introduction to dynamic meteorology (Vol. 88, 4th ed.). Elsevier Academic Press.

Horvat, C., \& Tziperman, E. (2017). The evolution of scaling laws in the sea ice floe size distribution. Journal of Geophysical Research: Oceans, 122(9), 7630-7650. https://doi.org/10.1002/2016JC012573

Huber, P. J. (1981). Robust statistics. John Wiley \& Sons.

Hunke, E. C. (2010). Thickness sensitivities in the CICE sea ice model. Ocean Modelling, 34(3-4), 137-149. https://doi.org/10.1016/j. ocemod.2010.05.004

Hunke, E. C., Allard, R., Bailey, D. A., Blain, P., Craig, A., Dupont, F., et al. (2020). CICE-Consortium/CICE: CICE version 6.1.2. Zenodo. https://doi.org/10.5281/zenodo.3888653

Hunke, E. C., \& Dukowicz, J. K. (2003). The sea ice momentum equation in the free drift regime (Technical Report No. LA-UR-03-2219). Los Alamos National Laboratory.

Hunkins, K. (1966). Ekman drift currents in the Arctic Ocean. Deep Sea Research and Oceanographic Abstracts, 13(4), 607-620. https://doi. org/10.1016/0011-7471(66)90592-4

Ivanov, V., Alexeev, V., Koldunov, N. V., Repina, I., Sandø, A. B., Smedsrud, L. H., \& Smirnov, A. (2016). Arctic Ocean heat impact on regional ice decay: A suggested positive feedback. Journal of Physical Oceanography, 46(5), 1437-1456. https://doi.org/10.1175/JPO-D-15-0144.1

Jackson, J. M., Allen, S. E., McLaughlin, F. A., Woodgate, R. A., \& Carmack, E. C. (2011). Changes to the near-surface waters in the Canada Basin, Arctic Ocean from 1993-2009: A basin in transition. Journal of Geophysical Research, 116(C10). https://doi. org/10.1029/2011JC007069

Johannessen, O. M. (1970). Note on some vertical profiles below ice floes in the Gulf of St. Lawrence and near the North Pole. Journal of Geophysical Research, 75(15), 2857-2861. https://doi.org/10.1029/JC075i015p02857

Kim, J. G., Hunke, E. C., \& Lipscomb, W. H. (2006). Sensitivity analysis and parameter tuning scheme for global sea-ice modeling. Ocean Modelling, 14(1), 61-80. https://doi.org/10.1016/j.ocemod.2006.03.003

Kim, T. W., Ha, H. K., Wåhlin, A. K., Lee, S. H., Kim, C. S., Lee, J. H., \& Cho, Y. K. (2017). Is Ekman pumping responsible for the seasonal variation of warm circumpolar deep water in the Amundsen Sea? Continental Shelf Research, 132, 38-48. https://doi.org/10.1016/j. csr.2016.09.005

Kirillov, S., Babb, D., Dmitrenko, I., Landy, J., Lukovich, J., Ehn, J., et al. (2020). Atmospheric forcing drives the winter sea ice thickness asymmetry of Hudson Bay. Journal of Geophysical Research: Oceans, 125(2). https://doi.org/10.1029/2019JC015756

Kobayashi, S., Ota, Y., Harada, Y., Ebita, A., Moriya, M., Onoda, H., et al. (2015). The JRA-55 reanalysis: General specifications and basic characteristics. Journal of the Meteorological Society of Japan, 93(1), 5-48. https://doi.org/10.2151/jmsj.2015-001

Köberle, C., \& Gerdes, R. (2003). Mechanisms determining the variability of Arctic sea ice conditions and export. Journal of Climate, 16(17), 2843-2858. https://doi.org/10.1175/1520-0442(2003)016〈2843:MDTVOA $\rangle$ 2.0.CO;2

Krishfield, R. A., Proshutinsky, A., Tateyama, K., Williams, W. J., Carmack, E. C., McLaughlin, F. A., \& Timmermans, M.-L. (2014). Deterioration of perennial sea ice in the Beaufort Gyre from 2003 to 2012 and its impact on the oceanic freshwater cycle. Journal of Geophysical Research: Oceans, 119(2), 1271-1305. https://doi.org/10.1002/2013JC008999

Large, W. G., \& Yeager, S. G. (2004). Diurnal to decadal global forcing for ocean and sea-ice models: The data sets and flux climatologies (Technical Note Nos. NCAR/TN-460+STR). National Center for Atmospheric Research.

Losch, M., Menemenlis, D., Campin, J.-M., Heimbach, P., \& Hill, C. (2010). On the formulation of sea-ice models. Part 1: Effects of different solver implementations and parameterizations. Ocean Modelling, 33(1-2), 129-144. https://doi.org/10.1016/j.ocemod.2009.12.008

Lu, P., Li, Z., Cheng, B., \& Leppäranta, M. (2011). A parameterization of the ice-ocean drag coefficient. Journal of Geophysical Research, 116(C7), C07019. https://doi.org/10.1029/2010JC006878

Lüpkes, C., \& Birnbaum, G. (2005). 'Surface drag in the Arctic marginal sea-ice zone: A comparison of different parameterisation concepts'. Boundary-Layer Meteorology, 117(2), 179-211. https://doi.org/10.1007/s10546-005-1445-8 
Lüpkes, C., \& Gryanik, V. M. (2015). A stability-dependent parametrization of transfer coefficients for momentum and heat over polar sea ice to be used in climate models. Journal of Geophysical Research: Atmospheres, 120(2), 552-581. https://doi.org/10.1002/2014JD022418

Lüpkes, C., Gryanik, V. M., Hartmann, J., \& Andreas, E. L. (2012). A parametrization, based on sea ice morphology, of the neutral atmospheric drag coefficients for weather prediction and climate models. Journal of Geophysical Research, 117(D13). https://doi. org/10.1029/2012JD017630

Lüpkes, C., Gryanik, V. M., Rösel, A., Birnbaum, G., \& Kaleschke, L. (2013). Effect of sea ice morphology during Arctic summer on atmospheric drag coefficients used in climate models. Geophysical Research Letters, 40(2), 446-451. https://doi.org/10.1002/grl.50081

Magnell, B., Ivanov, L., \& Siegel, E. (2010). Measurements of ice parameters in the Beaufort Sea using the Nortek AWAC acoustic Doppler current profiler. In OCEANS 2010 MTS/IEEE SEATTLE (pp. 1-8). IEEE. https://doi.org/10.1109/OCEANS.2010.5664016

Martin, T. (2007). Arctic sea ice dynamics: Drift and ridging in numerical models and observations (Unpublished doctoral dissertation). University of Bremen

Martin, T., Steele, M., \& Zhang, J. (2014). Seasonality and long-term trend of Arctic Ocean surface stress in a model. Journal of Geophysical Research: Oceans, 119(3), 1723-1738. https://doi.org/10.1002/2013JC009425

Martin, T., Tsamados, M., Schroeder, D., \& Feltham, D. L. (2016). The impact of variable sea ice roughness on changes in Arctic Ocean surface stress: A model study. Journal of Geophysical Research: Oceans, 121(3), 1931-1952. https://doi.org/10.1002/2015JC011186

Maykut, G. A., \& Untersteiner, N. (1971). Some results from a time-dependent thermodynamic model of sea ice. Journal of Geophysical Research, 76(6), 1550-1575. https://doi.org/10.1029/JC076i006p01550

McPhee, M. G. (1979). The effect of the oceanic boundary layer on the mean drift of pack ice: Application of a simple model. Journal of Physical Oceanography, 9(2), 388-400. https://doi.org/10.1175/1520-0485(1979)009〈0388:TEOTOB $2.0 . C O ; 2$

McPhee, M. G. (1980). An analysis of pack ice drift in summer. Sea ice processes and models, 62-75.

McPhee, M. G. (2002). Turbulent stress at the ice/ocean interface and bottom surface hydraulic roughness during the SHEBA drift. Journal of Geophysical Research, 107(C10), 8037. https://doi.org/10.1029/2000JC000633

McPhee, M. G. (2008). Air-ice-ocean interaction: Turbulent ocean boundary layer exchange processes. Springer-Verlag. https://doi. org/10.1007/978-0-387-78335-2

McPhee, M. G. (2012). Advances in understanding ice-ocean stress during and since AIDJEX. Cold Regions Science and Technology, 76-77, 24-36. https://doi.org/10.1016/j.coldregions.2011.05.001

McPhee, M. G., \& Kantha, L. H. (1989). Generation of internal waves by sea ice. Journal of Geophysical Research, 94(C3), 3287. https://doi. org/10.1029/JC094iC03p03287

McPhee, M. G., Kottmeier, C., \& Morison, J. H. (1999). Ocean heat flux in the central Weddell Sea during winter. Journal of Physical Oceanography, 29(6), 1166-1179. https://doi.org/10.1175/1520-0485(1999)029〈1166:OHFITC $2.0 . C O ; 2$

Meneghello, G., Marshall, J., Campin, J. M., Doddridge, E., \& Timmermans, M. L. (2018). The ice-ocean governor: Ice-ocean stress feedback limits Beaufort Gyre spin-up. Geophysical Research Letters, 45(20). 11293-11299. https://doi.org/10.1029/2018GL080171

Monin, A. S., \& Obukhov, A. M. (1954). Basic laws of turbulent mixing in the surface layer of the atmosphere. Trudy Geofiz Instituta Akademii Nauk SSSR, 24(151), 163-187.

Morison, J. H., McPhee, M. G., \& Maykut, G. A. (1987). Boundary layer, upper ocean, and ice observations in the Greenland Sea marginal ice zone. Journal of Geophysical Research, 92(C7), 6987-7011. https://doi.org/10.1029/JC092iC07p06987

Park, H.-S., \& Stewart, A. L. (2016). An analytical model for wind-driven Arctic summer sea ice drift. The Cryosphere, 10(1), 227-244. https://doi.org/10.5194/tc-10-227-2016

Perrie, W., \& Hu, Y. (1997). Air-ice-ocean momentum exchange. Part II: Ice drift. Journal of Physical Oceanography, 27(9), 1976-1996. https://doi.org/10.1175/1520-0485(1997)027<1976:aiomep>2.0.co;2

Petty, A. A., Tsamados, M. C., \& Kurtz, N. T. (2017). Atmospheric form drag coefficients over Arctic sea ice using remotely sensed ice topography data, spring 2009-2015. Journal of Geophysical Research: Earth Surface, 122(8), 1472-1490. https://doi.org/10.1002/2017JF004209

Phillips, O. M. (1985). Spectral and statistical properties of the equilibrium range in wind-generated gravity waves. Journal of Fluid Mechanics, 156(1), 505-531. https://doi.org/10.1017/S0022112085002221

Pite, H. D., Topham, D. R., \& van Hardenberg, B. J. (1995). Laboratory measurements of the drag force on a family of two-dimensional ice keel models in a two-layer flow. Journal of Physical Oceanography, 25(12), 3008-3031. https://doi. org/10.1175/1520-0485(1995)025〈3008:LMOTDF〉2.0.CO;2

Plueddemann, A. J., Krishfield, R., Takizawa, T., Hatakeyama, K., \& Honjo, S. (1998). Upper ocean velocities in the Beaufort Gyre. Geophysical Research Letters, 25(2), 183-186. https://doi.org/10.1029/97GL53638

Rainville, L., Lee, C., \& Woodgate, R. (2011). Impact of wind-driven mixing in the Arctic Ocean. Oceanography, 24(3), 136-145. https:// doi.org/10.5670/oceanog.2011.65

Rainville, L., \& Woodgate, R. A. (2009). Observations of internal wave generation in the seasonally ice-free Arctic. Geophysical Research Letters, 36(23), L23604. https://doi.org/10.1029/2009GL041291

Rampal, P., Bouillon, S., Ólason, E., \& Morlighem, M. (2016). neXtSIM: A new Lagrangian sea ice model. The Cryosphere, 10(3), 1055-1073. https://doi.org/10.5194/tc-10-1055-2016

Randelhoff, A., Sundfjord, A., \& Renner, A. H. H. (2014). Effects of a shallow pycnocline and surface meltwater on sea ice-ocean drag and turbulent heat flux. Journal of Physical Oceanography, 44(8), 2176-2190. https://doi.org/10.1175/JPO-D-13-0231.1

Roach, L. A., Horvat, C., Dean, S. M., \& Bitz, C. M. (2018). An emergent sea ice floe size distribution in a global coupled ocean-sea ice model. Journal of Geophysical Research: Oceans, 123(6), 4322-4337. https://doi.org/10.1029/2017JC013692

Roberts, A. F., Hunke, E. C., Kamal, S. M., Lipscomb, W. H., Horvat, C., \& Maslowski, W. (2019). A variational method for sea ice ridging in earth system models. Journal of Advances in Modeling Earth Systems, 11(3), 771-805. https://doi.org/10.1029/2018MS001395

Rousset, C., Vancoppenolle, M., Madec, G., Fichefet, T., Flavoni, S., Barthélemy, A., et al. (2015). The Louvain-La-Neuve sea ice model LIM3.6: Global and regional capabilities. Geoscientific Model Development, 8(10), 2991-3005. https://doi.org/10.5194/gmd-8-2991-2015

Shaw, W. J., Stanton, T. P., McPhee, M. G., \& Kikuchi, T. (2008). Estimates of surface roughness length in heterogeneous under-ice boundary layers. Journal of Geophysical Research, 113(C8), C08030. https://doi.org/10.1029/2007JC004550

Shcherbina, A., D’Asaro, E. A., Light, B., Deming, J. W., \& Rehm, E. (2016). Maiden voyage of the under-ice float. American Geophysical Union.

Shirasawa, K., \& Ingram, R. G. (1991). Characteristics of the turbulent oceanic boundary layer under sea ice. Part 1: A review of the iceocean boundary layer. Journal of Marine Systems, 2(1), 153-160. https://doi.org/10.1016/0924-7963(91)90021-L

Shirasawa, K., Ingram, R. G., \& Aota, M. (1989). Measurements in the boundary layer under landfast ice in the southeast Hudson Bay, Canada. Low Temperature Science, A(47), 213-221. 
Spreen, G., Kaleschke, L., \& Heygster, G. (2008). Sea ice remote sensing using AMSR-E 89-GHz channels. Journal of Geophysical Research, 113(C2). https://doi.org/10.1029/2005JC003384

Steele, M., Morison, J. H., \& Untersteiner, N. (1989). The partition of air-ice-ocean momentum exchange as a function of ice concentration, floe size, and draft. Journal of Geophysical Research, 94(C9), 12739. https://doi.org/10.1029/JC094iC09p12739

Steele, M., Zhang, J., Rothrock, D., \& Stern, H. (1997). The force balance of sea ice in a numerical model of the Arctic Ocean. Journal of Geophysical Research, 102(C9), 21061-21079. https://doi.org/10.1029/97JC01454

Steiner, N. (2001). Introduction of variable drag coefficients into sea-ice models. Annals of Glaciology, 33, 181-186. https://doi. org/10.3189/172756401781818149

Stern, H. L., Schweiger, A. J., Zhang, J., \& Steele, M. (2018). On reconciling disparate studies of the sea-ice floe size distribution. Elementa: Science of the Anthropocene, 6(1), 49. https://doi.org/10.1525/elementa.304

Stroeve, J., \& Notz, D. (2018). Changing state of Arctic sea ice across all seasons. Environmental Research Letters, 13(10), 103001. https:// doi.org/10.1088/1748-9326/aade56

Thomson, J., Lund, B., Hargrove, J., Smith, M. M., Horstmann, J., \& MacKinnon, J. A. (2021). Wave-driven flow along a compact marginal ice zone. Geophysical Research Letters, 48(3), e2020GL090735. https://doi.org/10.1029/2020GL090735

Timco, G. W., \& Burden, R. P. (1997). An analysis of the shapes of sea ice ridges. Cold Regions Science and Technology, 25(1), 65-77. https:// doi.org/10.1016/S0165-232X(96)00017-1

Timmermann, R., Danilov, S., Schröter, J., Böning, C., Sidorenko, D., \& Rollenhagen, K. (2009). Ocean circulation and sea ice distribution in a finite element global sea ice-ocean model. Ocean Modelling, 27(3-4), 114-129. https://doi.org/10.1016/j.ocemod.2008.10.009

Timmermans, M.-L., Toole, J., \& Krishfield, R. (2018). Warming of the interior Arctic Ocean linked to sea ice losses at the basin margins. Science Advances, 4(8), eaat6773. https://doi.org/10.1126/sciadv.aat6773

Tsamados, M., Feltham, D. L., Schroeder, D., Flocco, D., Farrell, S. L., Kurtz, N., et al. (2014). Impact of variable atmospheric and oceanic form drag on simulations of Arctic sea ice. Journal of Physical Oceanography, 44(5), 1329-1353. https://doi.org/10.1175/JPO-D-13-0215.1

Wadhams, P., \& Davy, T. (1986). On the spacing and draft distributions for pressure ridge keels. Journal of Geophysical Research, 91(C9), 10697-10708. https://doi.org/10.1029/JC091iC09p10697

Wadhams, P., \& Horne, R. J. (1980). An analysis of ice profiles obtained by submarine sonar in the Beaufort Sea. Journal of Glaciology, 25(93), 401-424. https://doi.org/10.3189/S0022143000015264

Weiss, J., \& Marsan, D. (2004). Scale properties of sea ice deformation and fracturing. Comptes Rendus Physique, 5(7), 735-751. https://doi. org/10.1016/j.crhy.2004.09.005

Williams, E., Swithinbank, C., \& Robin, G. D. Q. (1975). A submarine sonar study of Arctic pack ice. Journal of Glaciology, 15(73), 349-362. https://doi.org/10.3189/S002214300003447X

$\mathrm{Zu}$, Y., Lu, P., Lepparanta, M., Cheng, B., \& Li, Z. (2020). On the form drag coefficient under ridged ice: Laboratory experiments and numerical simulations from ideal scaling to real ice conditions [Preprint]. Earth and Space Science Open Archive. https://doi.org/10.1002/ essoar.10504763.1 